\title{
Narrative review of epilepsy: getting the most out of your neuroimaging
}

\author{
Andrea De Vito ${ }^{1}$, Kshitij Mankad ${ }^{2}$, Suresh Pujar ${ }^{3}$, Aswin Chari ${ }^{4}$, Davide Ippolito ${ }^{5}$, Felice D'Arco ${ }^{2}$ \\ ${ }^{1}$ Department of Neuroradiology, H. S. Gerardo Monza, Monza, Italy; ${ }^{2}$ Department of Radiology, Great Ormond Street Hospital, London, UK; \\ ${ }^{3}$ Department of Neurology, Great Ormond Street Hospital for Children, London, UK; ${ }^{4}$ Department of Neurosurgery, Great Ormond Street Hospital, \\ London, UK; ${ }^{5}$ Department of Radiology, H. S. Gerardo Monza, Monza, Italy \\ Contributions: I) Conception and design: F D'Arco; (II) Administrative support: F D'Arco; (III) Provision of study materials or patients: F D'Arco; \\ (IV) Collection and assembly of data: A De Vito; (V) Data analysis and interpretation: A De Vito; (VI) Manuscript writing: All authors; (VII) Final \\ approval of manuscript: All authors. \\ Correspondence to: Andrea De Vito, MD. Department of Neuroradiology, H. S. Gerardo Monza, Via Pergolesi 33, 20900 Monza (MB), Italy. \\ Email: a.devito@campus.unimib.it.
}

\begin{abstract}
Neuroimaging represents an important step in the evaluation of pediatric epilepsy. The crucial role of brain imaging in the diagnosis, follow-up and presurgical assessment of patients with epilepsy is noted and has to be familiar to all neuroradiologists and trainees approaching pediatric brain imaging. Morphological qualitative imaging shows the majority of cerebral lesions/alterations underlying focal epilepsy and can highlight some features which are useful in the differential diagnosis of the different types of epilepsy. Recent advances in MRI acquisitions including diffusion-weighted imaging (DWI), postacquisition image processing techniques, and quantification of imaging data are increasing the accuracy of lesion detection during the last decades. Functional MRI (fMRI) can be really useful and helps to identify cortical eloquent areas that are essential for language, motor function, and memory, and diffusion tensor imaging (DTI) can reveal white matter tracts that are vital for these functions, thus reducing the risk of epilepsy surgery causing new morbidities. Also positron emission tomography (PET), single photon emission computed tomography (SPECT), simultaneous electroencephalogram (EEG) and fMRI, and electrical and magnetic source imaging can be used to assess the exact localization of epileptic foci and help in the design of intracranial EEG recording strategies. The main role of these "hybrid" techniques is to obtain quantitative and qualitative informations, a necessary step to evaluate and demonstrate the complex relationship between abnormal structural and functional data and to manage a "patient-tailored" surgical approach in epileptic patients.
\end{abstract}

Keywords: Central nervous system diseases; epilepsy; pre-surgical planning

Submitted Aug 23, 2020. Accepted for publication Nov 26, 2020.

doi: $10.21037 /$ tp-20-261

View this article at: http://dx.doi.org/10.21037/tp-20-261

\section{Introduction}

\section{Definition of epilepsy}

Epilepsy is a widespread neurologic disease, which does not represent an unique entity but rather a heterogeneous group of diverse pathologies reflecting the underlying brain dysfunction that may result from many different etiologies (1). It affects all ages of the population and its incidence in childhood is approximately $50-100$ cases per 100,000 persons per year with the highest incidence in the first year of life $(2,3)$. Higher mortality rates are seen in epileptic population, almost three times higher compared with the expected age and sex adjusted mortality in general population. Anti-epileptic drugs treatment has shown to be useful in a majority of epileptic patients, but unfortunately in almost $30 \%$ of patients the epileptic seizures are 
untreatable and recurrent (4). In non-responding epileptic patients, surgical approach may be helpful to remove the epileptic trigger and can be a promising alternative, with potential for improving seizure frequency, severity and quality of life. Indeed it is critical to identify an epileptogenic lesion on imaging and precisely define its margin, morphology and location.

\section{Clinical manifestations}

Epileptic seizures are clinical events and their presentation strongly depends on different factors such as the brain location of the epileptogenic focus, the maturity of the brain, the sleep-wake cycle and others elements (5). Signs and symptoms of the epileptic activity are important for the definition of epilepsy, but is often complicated to evaluate in detail the subjective and objective clinical phenomena of a seizure, due to the wide range of possible manifestations. Seizures can affect sensory, motor, and autonomic function; also, consciousness, emotional state, memory, cognition and behavior can be affected (6).

\section{Role of imaging}

MRI represents the "gold standard" imaging study used to reveal the pathological substrates of regional brain epileptogenesis, and, despite some cases remain idiopathic, the use of high field MRI dramatically changed the outcome in many children with epilepsy (7). Indeed, MRI plays a fundamental major role in the management of epileptic patients, helping neuroradiologists and clinicians localizing epileptogenic foci and in the pre-surgical assessment of patients who don't respond to drug therapy; MRI is also known to be the most adequate tool to follow-up drugresistant focal epilepsies, which constitute about $25 \%$ of epilepsy cases (8).

An MR study must always be performed in the nonemergency setting to evaluate all patients referred to our departments for epilepsy (except for generalized nonfocal epilepsy) (9-11). Regardless of the underlying disease, patients in whom the lesions are visualized at preoperative MRI tend to have a better outcome after surgery for epilepsy than do patients without lesions (12). Computed tomography (CT), which once had a role in the evaluation of epileptic patients, is now considered as a second level imaging approach and its main advantage is represented by the high sensitivity in the detection of calcifications, as seen in some phakomatoses (e.g., Sturge-Weber disease) or in some epileptogenic neoplasms (13). Every pediatric neuroradiologist should be aware of the clinical signs and symptoms of epilepsy, have some knowledge of electroencephalogram (EEG) interpretation and earn experience in the imaging assessment of epilepsy. This paper is conceived as a practical guide to modern neuroimaging in epilepsy for trainees and fellows.

The key questions and objectives of this review are to summarize and explain the physiopathological mechanisms underlying the main pediatric epilepsy etiologies and to briefly approach new emerging imaging techniques useful in the pre-surgical management of pediatric epileptic patients. We present the following article in accordance with the Narrative Review reporting checklist (available at http:// dx.doi.org/10.21037/tp-20-261).

\section{Methods}

Spanning the last 20 years, multiple epilepsy papers have been published describing neuroimaging manifestations, pathophysiology and multidisciplinary approach to pediatric epilepsy. Our search strategy on PubMed, developed in consultation with a librarian with expertise in health research and systematic reviews, included terms related to epilepsy and pediatric epilepsy (in studies published from 1995). A broad approach was taken through the inclusion of all possible synonyms and abbreviations for the terms of interest, and controlled vocabulary/subject headings (including MeSH, EmTree).

\section{Discussion}

\section{Technical aspects of modern neuroimaging in epilepsy}

\section{T vs. $1.5 \mathrm{~T}$}

High field scanner such as 3 Tesla (T) MR represents the "gold standard" for epilepsy imaging (14). Compared to the standard $1.5 \mathrm{~T}$ scanner, the $3 \mathrm{~T} \mathrm{MR}$ provides a higher signal strength relative to the background noise [signal-to-noise (SNR) ratio], higher resolution imaging and seems to have higher sensitivity identifying focal lesions, especially the subtle ones which are frequently misdiagnosed on MRI (15) (Figure 1). There is no class I evidence demonstrating the superiority of $3 \mathrm{~T}$ MRI relative to $1.5 \mathrm{~T}$ MRI. However, some authors comparing $3 \mathrm{~T}$ with $1.5 \mathrm{~T} \mathrm{MR}$ in patients with no lesion or ambiguous findings on $1.5 \mathrm{~T}$ suggested that $3 \mathrm{~T}$ has higher capacity of identifying a lesion $(16,17)$. Knake et al. reported that the findings from $3 \mathrm{~T}$ MRI 

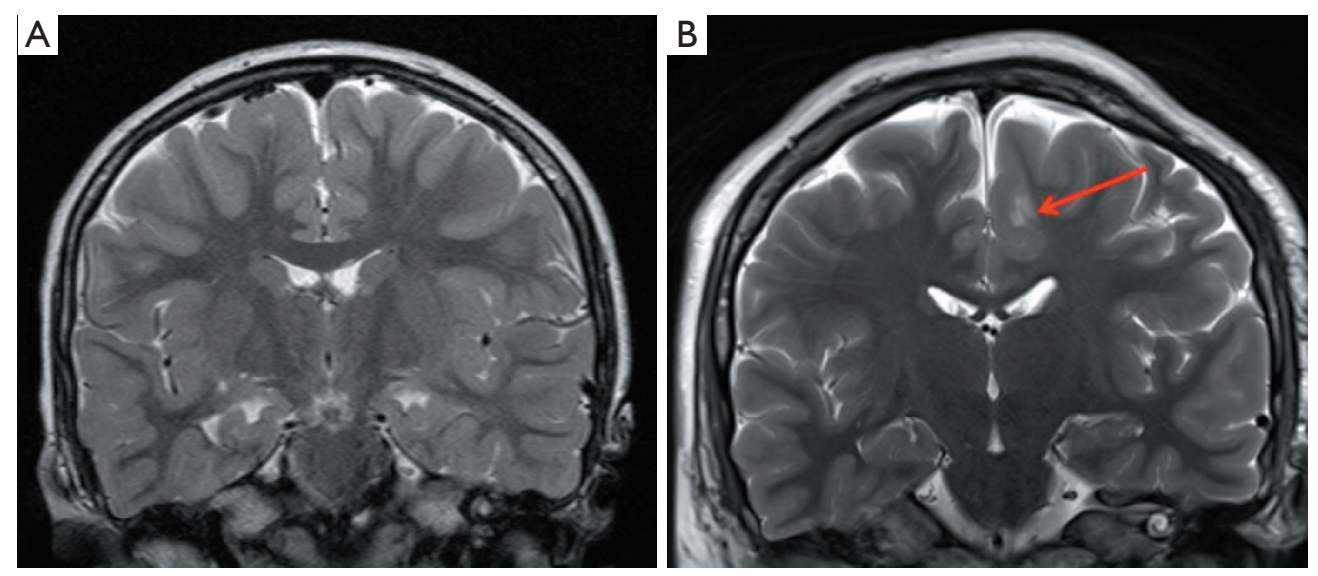

Figure 1 Coronal T2-WI from two different brain MRI scans, respectively performed on 1.5T (A) and 3T (B) scan, showing focal blurring of the white-grey matter interface (red arrow) in the left parietal lobe. T2-WI, T2-weighted images.
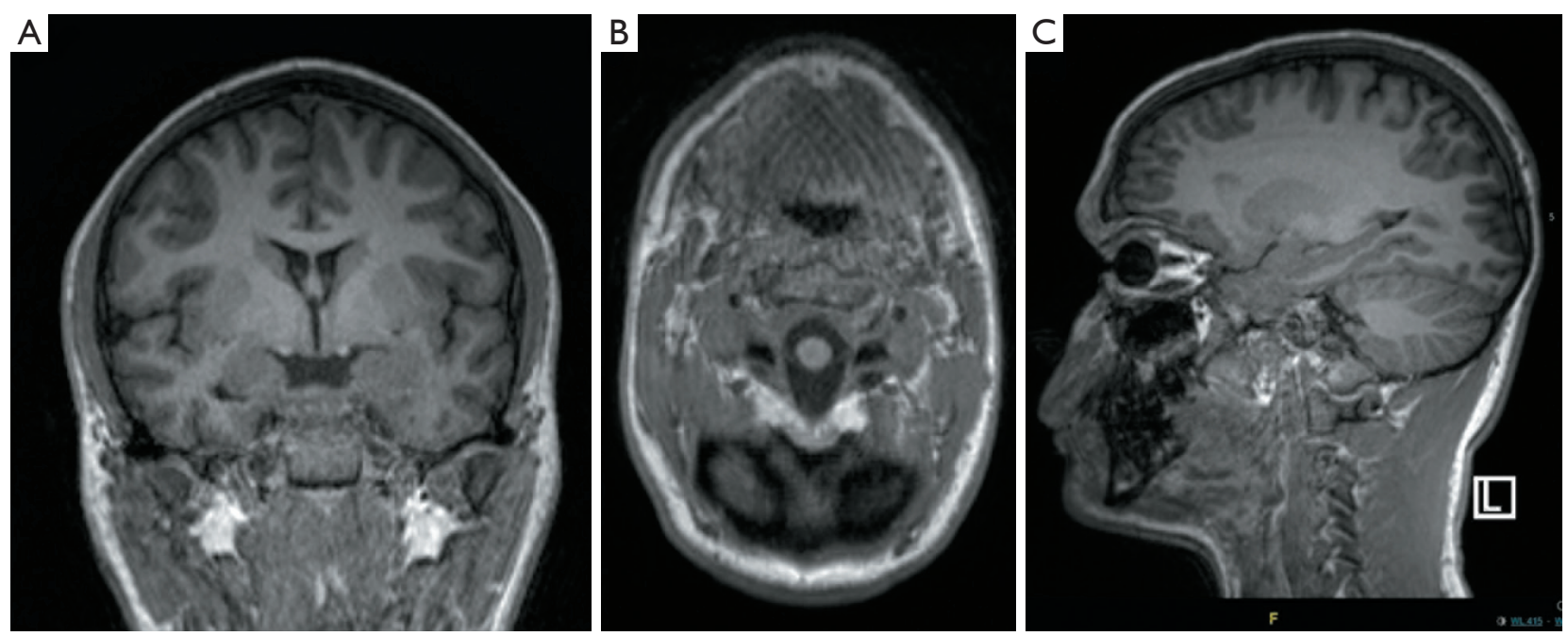

Figure 2 Coronal (A), axial (B) and sagittal (C) reformats from a 3D T1 sequence performed on a $3 \mathrm{~T}$ scan, showing its high sensitivity to motion artifacts.

changed clinical management in 8 of 15 cases, including omission of invasive monitoring, defining a clear target for invasive monitoring, and reducing the region requiring invasive monitoring. Rubinger et al. (18) have shown that improvement in structural imaging through the use of high-resolution epilepsy protocol on 3T MRI can increase lesion identification and also improve seizure free epilepsy surgery outcome. However, 3T scanner is extremely sensitive to flow and motion artifacts (in particular affecting temporal regions) and need expert set-up of the sequences and low threshold for scan under GA or deep sedation in epilepsy patients; it is important to remember that three- dimensional (3D) acquisitions with reformats are crucial in epilepsy imaging for their high spatial resolution but if motion artifacts are present in the original sequence they will be also visible in the reformats (Figure 2). Regular quality control of the MRI scanner is necessary to minimize artifacts.

\section{Suggested MRI protocol}

The main characteristics of a "patient-tailored" imaging protocol in epilepsy studies should be providing optimal volumes for visual assessment of the brain and at the 


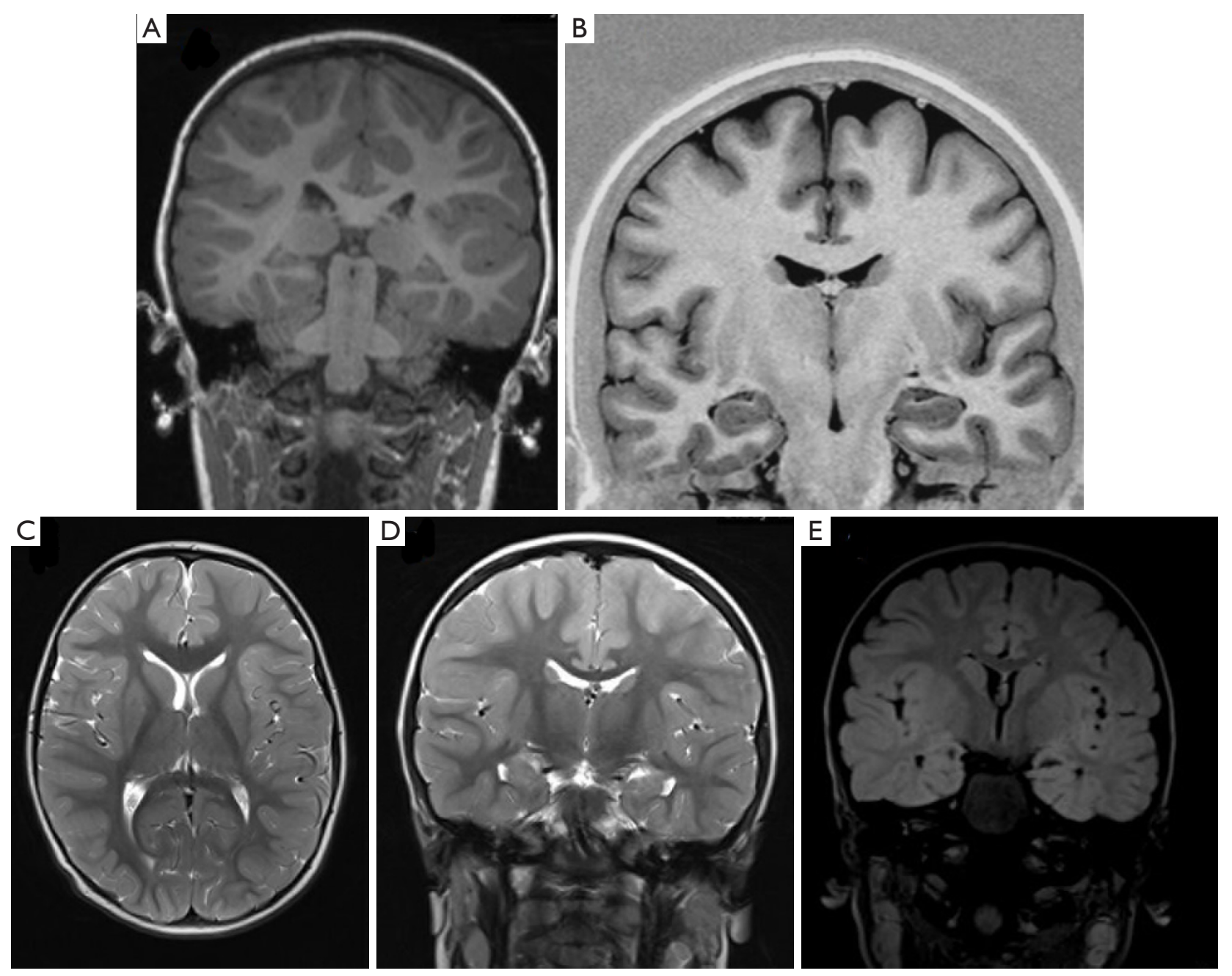

Figure 3 Suggested epilepsy MRI protocol standard sequences: (A) 3D T1 sequence, (B) 3D T1-IR sequence, (C) 2D T2 axial sequence, (D) 2D T2 coronal sequence, (E) 3D FLAIR sequence. T1-IR, T1-inversion recovery; FLAIR, fluid-attenuated inversion recovery sequence.

same time supplying ideal input data for post-processing. Epileptic patients undergoing MRI can be categorized into two big sub-groups, based on the different locations of the epileptic foci, respectively in the temporal lobe or in extratemporal regions (19). Temporal lobe epilepsy represents an electro clinically homogenous group and are less frequent in children when compared to adults where they represent the largest subgroup. For both groups, 3D T1-weighted images (WI) and T1-inversion recovery (IR) and isotropic 3D fluid-attenuated inversion recovery sequence (FLAIR) are pivotal sequences. These sequences should be run first, as they can be used to plan angulation of the following $2 \mathrm{D}$ sequences $(20,21)$ (Figure 3).

* High-resolution 3D T1-weighted (T1-w) and T1-
IR: the magnetization-prepared rapid gradient-echo (MP-RAGE) sequence, as well as the equivalent 3D spoiled gradient echo and $3 \mathrm{D}$ turbo field echo protocols with isotropic millimetric voxel resolution (i.e., $1 \times 1 \times 1 \mathrm{~mm}^{3}$ ) are the most popular 3D T1-w GE sequences (21). Their main role is to provide the most appropriate images to evaluate brain anatomy.

* High-resolution 3D FLAIR: the main role of this $3 \mathrm{D}$ FLAIR is to assess signal abnormalities, especially white matter hyperintensities, tumors and other pathological alterations. In FLAIR sequences the suppression of cerebrospinal fluid (CSF) signal highlights the distinctness of 
hyperintense periventricular or cortical lesions, in comparison with standard T2-w sequences. This sequence should also be acquired with isotropic millimetric voxel resolution (i.e., $1 \times 1 \times 1 \mathrm{~mm}^{3}$ ) and no interslice gap. Due to the immaturity of white matter myelination, FLAIR images are less sensitive to epileptogenic pathology in infants (0-2 years) (22). In addition to the $3 \mathrm{D}$ sequences we suggest axial and coronal 2D T2 spin echo sequences with $2 \mathrm{~mm}$

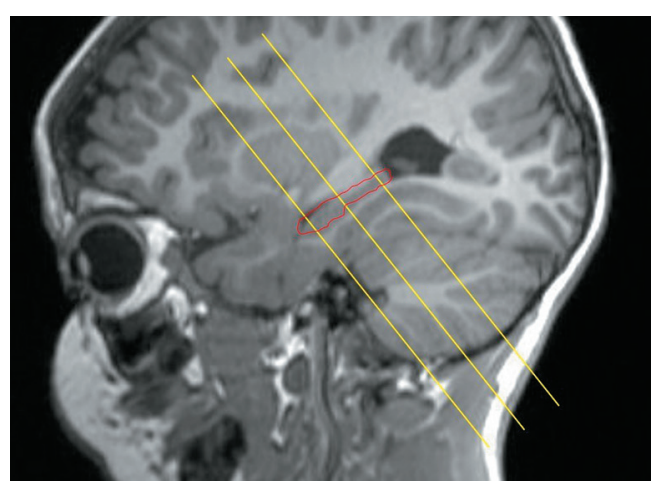

Figure 4 Sagittal T1-WI showing the right inclination of the coronal sequences perpendicularly to the hippocampal axis for the evaluation of HS. T1-WI, T1-weighted images; HS, hippocampal sclerosis. slice thickness. Some institutions run a 3D T2-WI sequence instead and/or 2D proton density as well. In cases of temporal lobe epilepsy patients, coronal slices should be angulated perpendicular to the hippocampal long axis to allow better identification of subtle hippocampal sclerosis (HS) (23) (Figure 4). 3D, isotropic volumes allows surface rendering of the brain that can be used as reference by the neurosurgeon (i.e., surface anatomy similar to the operative view); furthermore, for automated post processing, 3D isotropic volumes are also a prerequisite, because individual volumes have to be normalized to a template volume for inter-subject comparison (24) (Figure 5). Optional sequences are: SWI [for calcified tumors, in case of suspected Sturge-Weber syndrome (SWS) (25)] or tuberous sclerosis complex (TSC), post-contrast sequences for tumors, diffusion tensor imaging (DTI) and functional MRI (fMRI) for pre-operative planning, perfusion techniques such as arterial spin labeling (ASL) which provides useful information about vascularization and vascular proliferation, which is directly applicable to the assessment of brain tumors (26). In case of SWS or any situation where leptomeningeal enhancement is suspected we suggest adding post-contrast FLAIR which

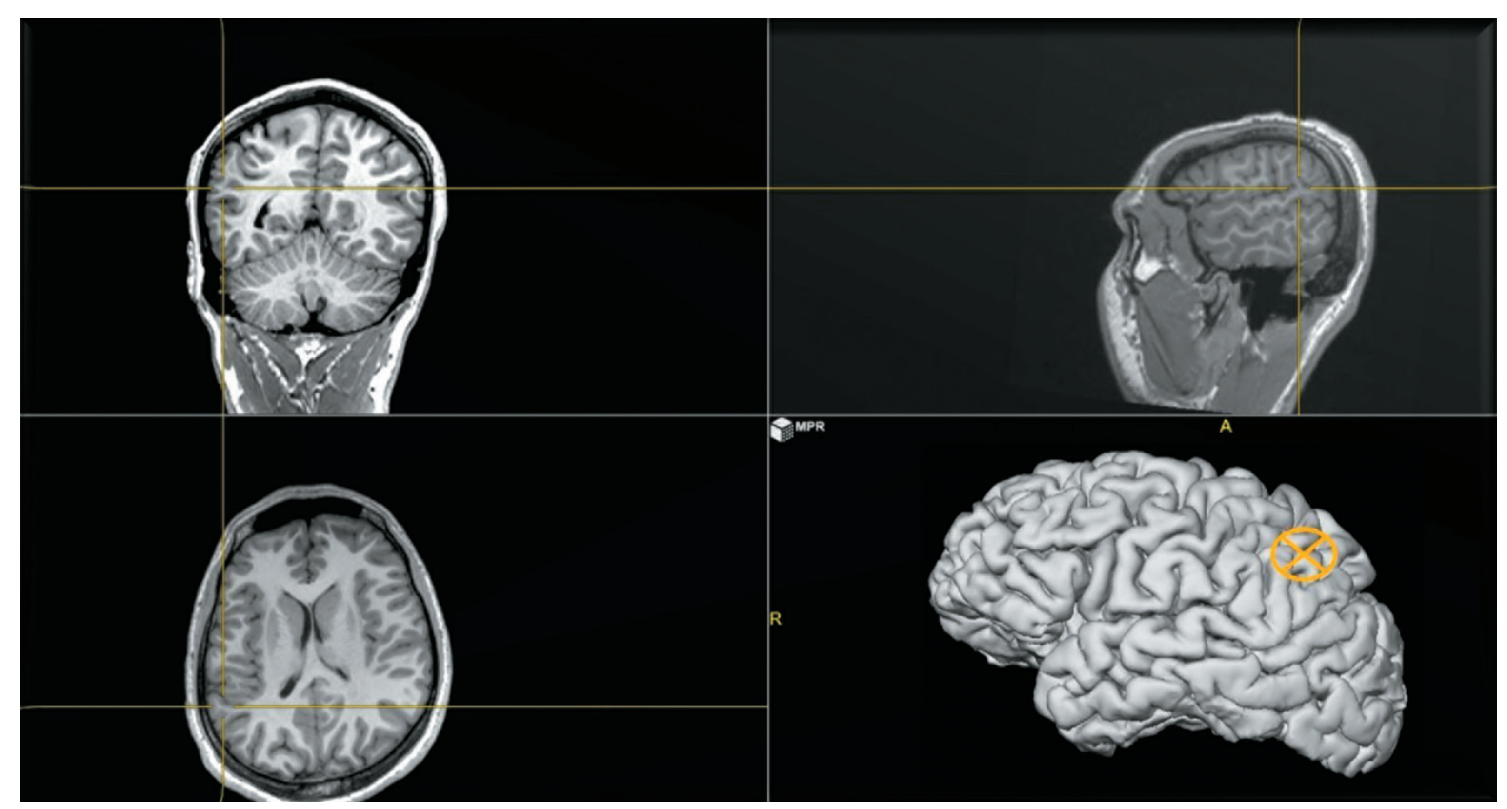

Figure 5 MPR and volume rendering formats in the assessment and pre-surgical evaluation of FCD. MPR, multiplanar reformations; FCD, focal cortical dysplasia. 

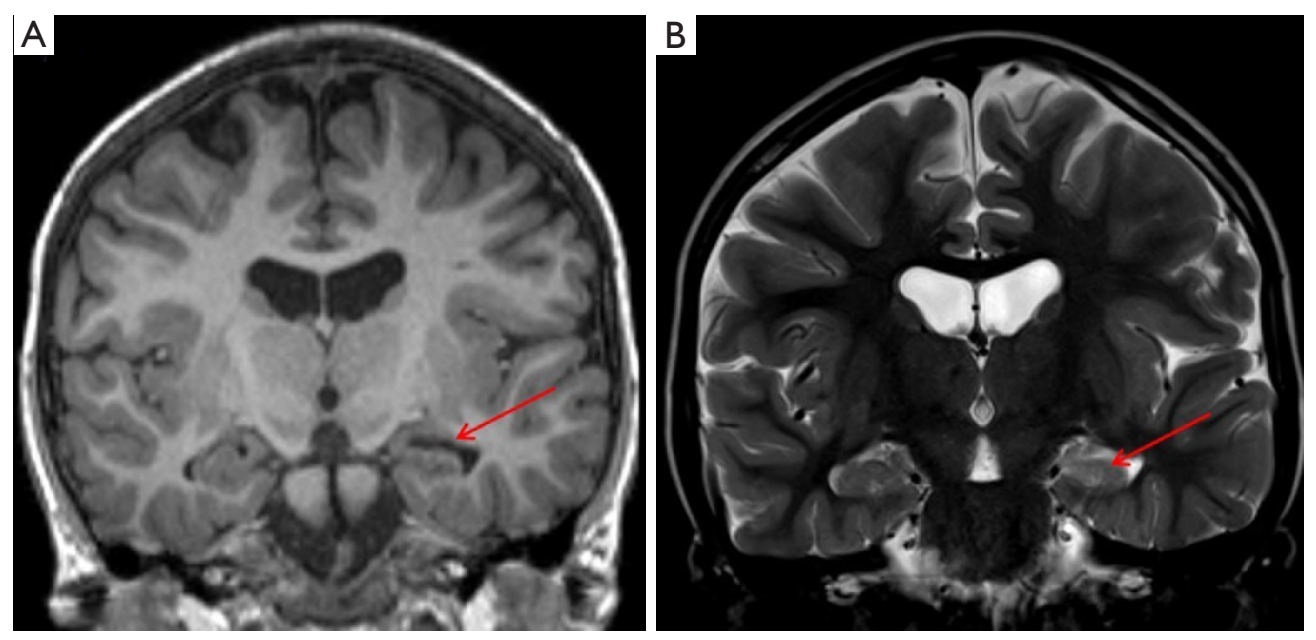

Figure 6 Coronal T1-w (A) and T2-w (B) sequences showing a small hippocampus with bright signal in T2-WI on the left side (red arrows). T1-w, T1-weighted; T2-w, T2-weighted; T2-WI, T2-weighted images.

has the highest sensitivity to subtle superficial enhancement.

Children (1-18 years): (I) volumetric T1-w and IR T1$\mathrm{w}$ : isotropic voxels, $1 \mathrm{~mm}$ with optimal grey-white matter contrast and with multiplanar reformations (MPR). (II) 3D FLAIR: slice thickness $1 \mathrm{~mm}$. (III) Axial turbo-spin-echo T2-WI: slice thickness of 2-V3 mm, gap 0-0.5 mm, whole brain coverage. (IV) diffusion-weighted imaging (DWI) axial b0-b1000 4 mm. (V) Optional: SWI, DTI, fMRI, ASL and post-contrast sequences.

Neonates and infants ( $<1$ year): axial and coronal doubleecho short tau inversion recovery (DESTIR) are used instead of the standard T2-WI (however conventional 2D T2-w sequences can be used if DESTIR is not available) (27).

\section{MRI of epileptogenic substrates}

Focal epilepsy can be categorized into the following main five groups: (I) HS, (II) malformations of cortical development (MCD), (III) neoplasms, (IV) vascular abnormalities, (V) miscellaneous. Each substrate can be defined by some diverse imaging features which are useful to the radiologist in the differential diagnosis of the different pathologies (28).

\section{Mesiotemporal sclerosis}

Mesiotemporal sclerosis is by far on of the most common diagnosis made in case of adult population epilepsy; it represents only $20 \%$ of pediatric epilepsy (29). Mesial temporal sclerosis (MTS) can be seen as early as 14 months (30) and has been recognized by Dunleavy et al. (31) in some neonatal murine models. The most striking imaging findings of mesiotemporal sclerosis are represented by hippocampal atrophy and hyperintense signal in $\mathrm{T} 2-\mathrm{w}$ sequences, which reflect the histopathological changes in MTS (predominantly characterized by gliosis and neuronal loss). Apart from these, there are some more minor imaging features in MTS from volume loss of the temporal lobe to dilatation of the temporal horn of the lateral ventricle and atrophy of the mammillary bodies (32) (Figure 6). Usually the hippocampal formation is not uniformly affected, with the dentate gyrus, and the CA1, CA4 and to a lesser degree CA3 sections of the hippocampus being primarily involved (33). Although the dentate gyrus, the CA1, CA4 and to a lesser degree CA3 sections of the hippocampus are primarily involved, regional involvement may be difficult to characterize on clinical MRI exams and does not impact the clinical management of the patients. On MRI, coronal T2-w and FLAIR images are the most sensitive for detecting abnormal signal, while T1 3D can better define the loss of hippocampal gyration (34). Some authors (35) have reported that MR qualitative imaging have a sensitivity ranging from $80 \%$ to $90 \%$ in the evaluation of mesiotemporal sclerosis while using quantitative assessment the sensitivity rises to $90 \%$ to $95 \%$. Additional sequences like volumetry of hippocampus and T2-relaxometry may also be used for their potential role in helping lateralizing the epileptogenic focus (36).

\section{Hippocampal malrotation (HIMAL)}

HIMAL is characterized by incomplete inversion of the hippocampus with a rounded shape and blurred internal 

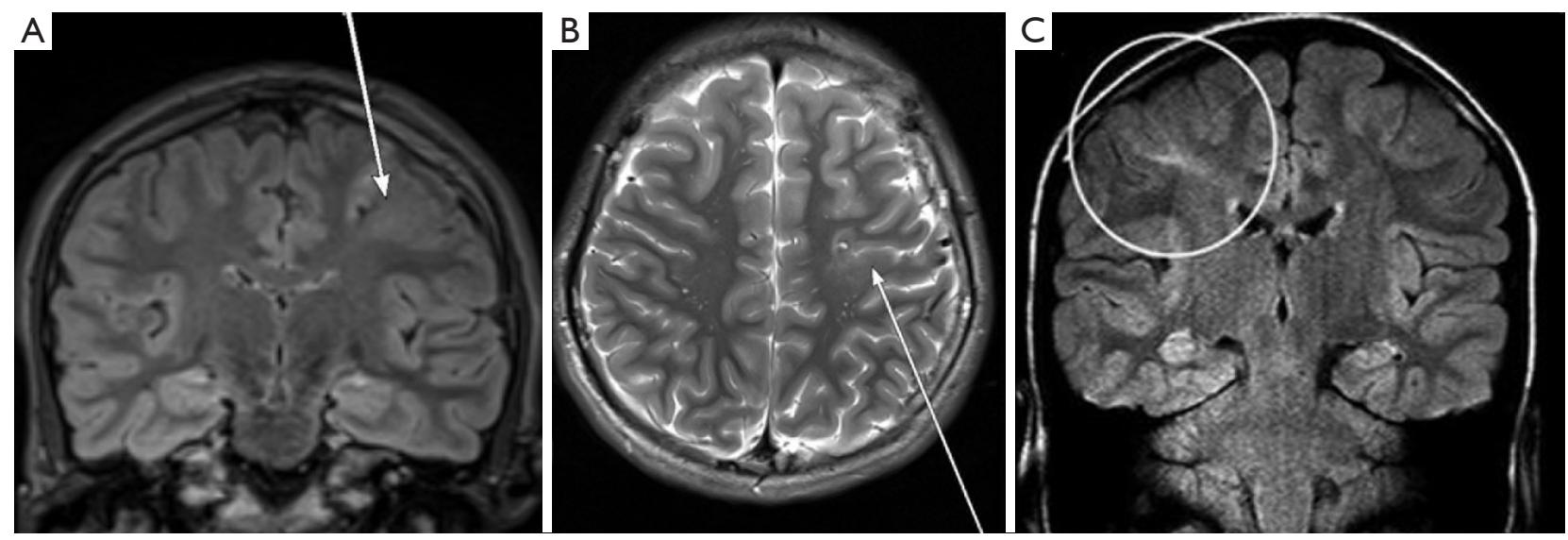

Figure 7 Coronal FLAIR (A) and axial 2D T2 (B) acquisition showing a left frontal sub-cortical hyperintense alteration compatible with "transmantle sign" (white arrows). Coronal FLAIR image (C) showing right parietal lobe "transmantle sign" (white circle). FLAIR, fluidattenuated inversion recovery sequence.

architecture, associated with a vertical collateral sulcus. Unlike HS, HIMAL is typically seen without evidence of abnormal signal intensity or visually evident volume loss of the hippocampus and has been reported as present more frequently on MRI examinations of patients with epilepsy, than those without epilepsy. Constellation of MRI findings include incomplete inversion of the hippocampus with an abnormally round shape (but with normal signal intensity and size), abnormal angle of the collateral sulcus, abnormal position and size of the fornix and enlargement and particular configuration of the temporal horn (37).

\section{Malformations of cortical development}

During the last decades, due to the increasing use of MRI in epilepsy, cortical malformations have been "reconsidered" as one of the most frequent etiology of focal epileptic seizures in both pediatric and young adults population. Indeed, from $10 \%$ to $50 \%$ of surgically resectable epileptic lesions in children are represented by MCD (only 4\% to $25 \%$ in adult population) (38). MCD have been categorized into four sub-categories, based on the patophysiological underlying mechanism: (I) abnormal neuronal and glial proliferation/apoptosis, (II) abnormal neuronal migration, (III) abnormal cortical organization and (IV) not otherwise classified (NOS) (39). A heterogeneous and diverse range of neuroimaging features have been strongly related to MCD, spanning from thickening of the cortex to blurring of grey/ white matter interface and from $\mathrm{T} 2-\mathrm{w}$ signal abnormalities in the juxtacortical areas to foci of heterotopic grey matter $(40,41)$.

\section{Focal cortical dysplasia (FCD)}

The most common malformation of cortical development encountered in epilepsy surgery is FCD and it results from perturbation in migration and/or cellular differentiation during gestation (42). Forty percent of children with focal intractable epilepsy has have a cortical malformation and its prevalence in surgical pediatric series is even higher, presenting clearly the predominant pathology (43). Cortical dysplasia can be focal, multifocal, or even hemispheric (hemimegalencephaly, although in these cases histology can be variable and include not only FCD), with the frontal and temporal lobes being the prevailing locations although FCD can occur anywhere (44). Moreover, areas of cortical dysplasia are better recognizable in both unmyelinated and fully myelinated brain but become less visible during the myelination period (4 months to 1.5 years). Main neuroimaging features of FCD:
* Thickening of the cortex;
* Blurring of white-grey matter junction with abnormal architecture of subcortical layer;
* White matter hyperintensity in T2/FLAIR sequences;
* T1 or T2 hyperintensity of the cortex;
* "Transmantle sign" (characteristic of the FCD type II-B) (Figure 7).

The most used classification of FCD is represented by Blumcke classification (45).

\section{Type I}

* Grey/white matter interface blurring (less marked than with type II FCD);

* Loss of white matter volume causing lobar atrophy; 

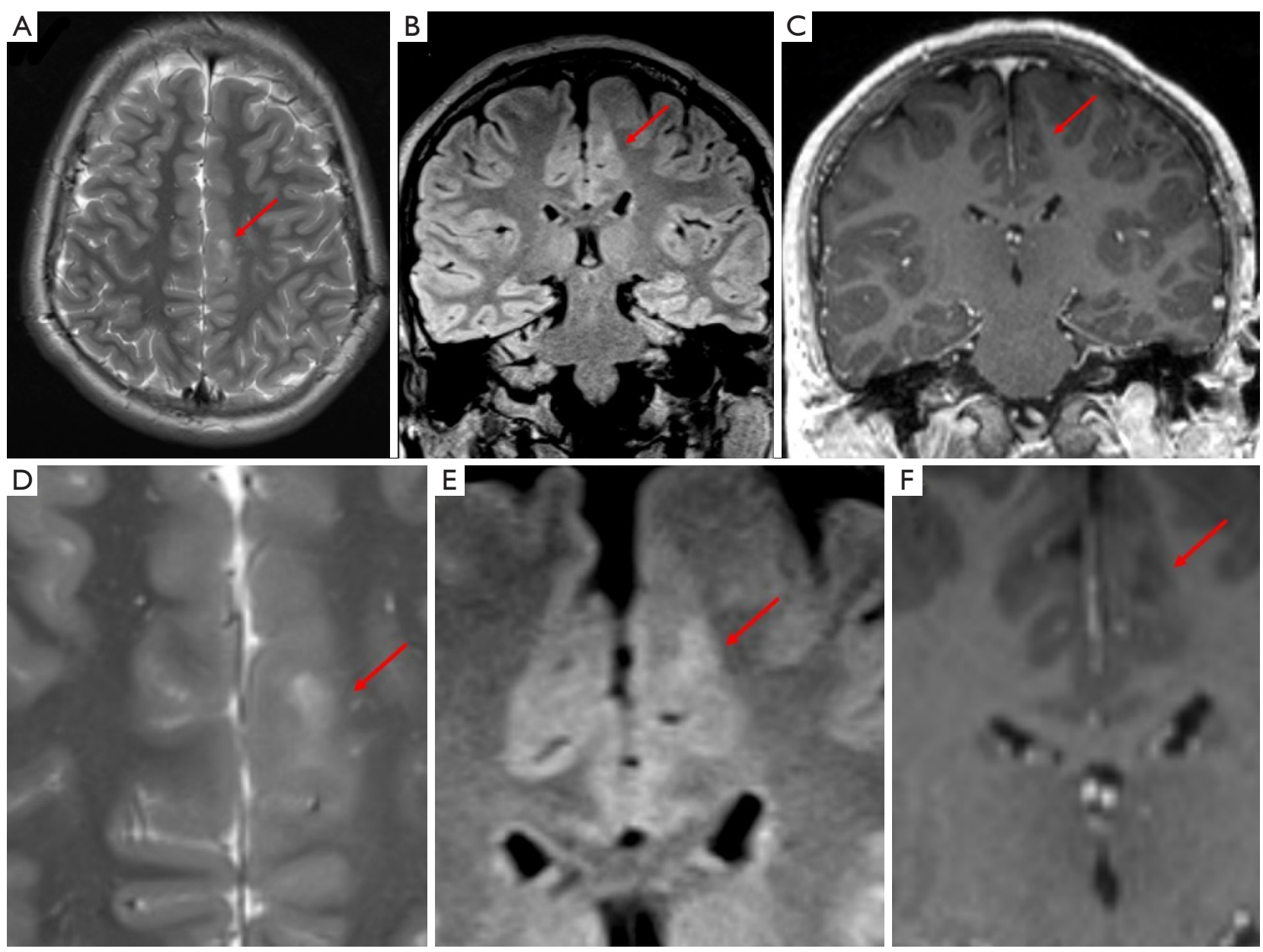

Figure 8 Axial T2-w (A), coronal FLAIR (B) and T1-w post-contrast (C) images showing a subtle area of cortical thickening and subcortical "blurring" in the left superior frontal gyrus (red arrows), better recognizable on the magnified images (D-F), compatible with FCD type II-B. T2-w, T2-weighted; FLAIR, fluid-attenuated inversion recovery sequence; T1-w, T1-weighted; FCD, focal cortical dysplasia.

* Hyperintense white matter signal in T2/FLAIR sequences and hypointense signal in $\mathrm{T} 1-\mathrm{w}$ sequences.

Note that FCD type I can be a difficult entity to diagnose histologically and there may be overlap with a not-well defined entity called oligodendrogliosis, which represents a spectrum of different oligodendroglial abnormalities, including oligodendroglial hyperplasia, clusters of oligodendroglia, oligodendroglial hamartoma and oligodendroglial-like cells without associated evidence of cortical dyslamination (46).

\section{Type II (Figure 8)}

* Frontal lobes represent the predominant location;

* Abnormal gyri and sulci;

* Grey/white matter junction blurring;
* Thickened cortex;

* White matter hyperintense signal in T2/FLAIR sequences, most striking and evident compared to type I;

* Sign.

\section{Type III}

A FCD is considered type III when found in association with other abnormalities in the adjacent parenchyma [e.g., IIIa-hippocampal atrophy; IIIb—glioneuronal tumor (e.g., DNET or ganglioglioma); IIIc—vascular malformation; IIId—early childhood insult (e.g., gliosis)].

- Frontal lobes represent the predominant location;

* Abnormal gyri and sulci;

* Grey/white matter junction blurring;

* Thickened cortex; 

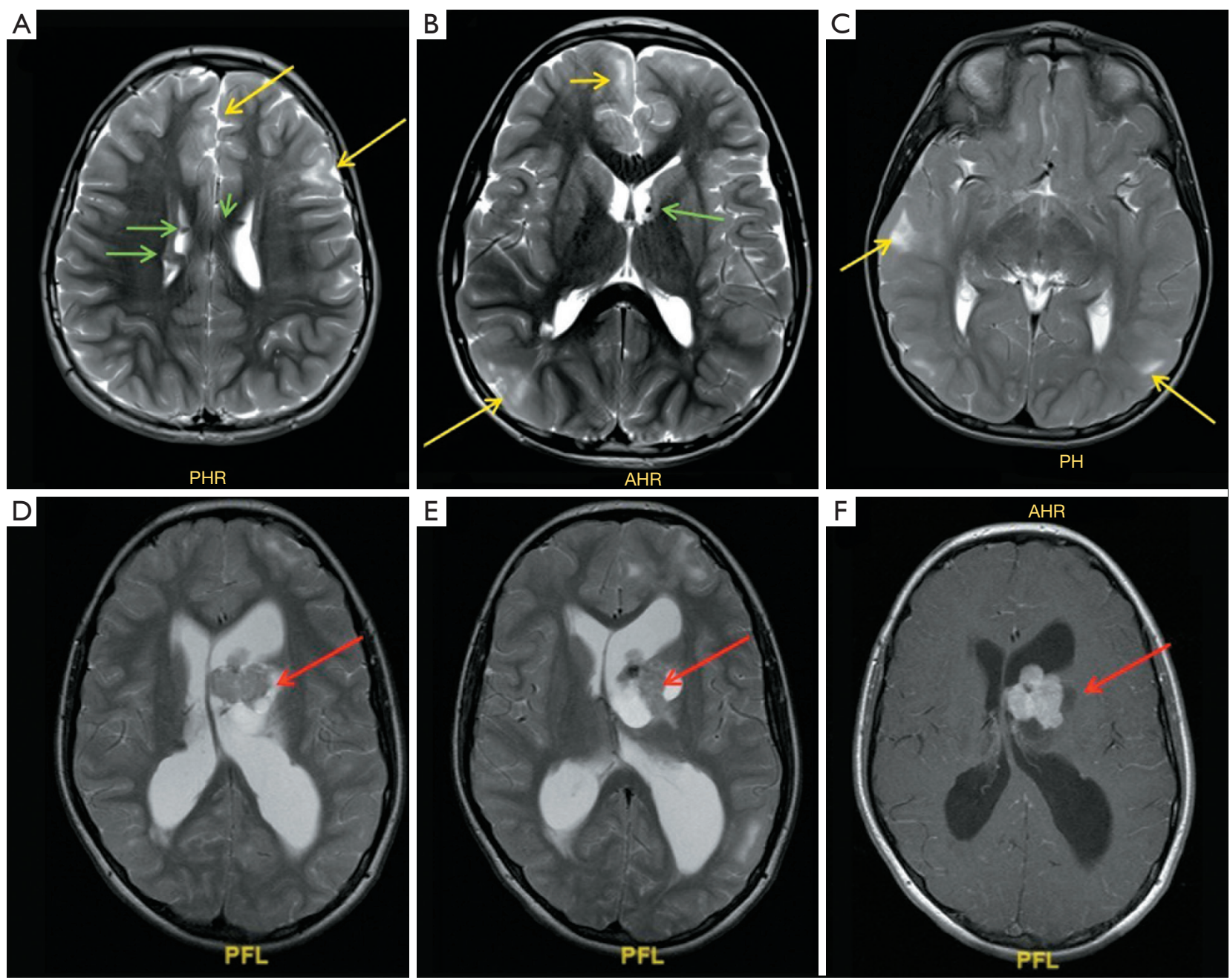

Figure 9 Axial T2-w (A-E) and T1-w post-contrast (F) images showing the presence of multiple cortical/subcortical tubers (yellow arrows), subependymal nodules (green arrows) and an enhancing SEGA (red arrows). The findings are compatible with TSC. T2-w, T2-weighted; T1-w, T1-weighted; SEGA, subependymal giant astrocytoma; TSC, tuberous sclerosis complex.

* White matter hyperintense signal in T2/FLAIR sequences, most striking and evident compared to type I;

* Transmantle sign.

\section{Tuberous sclerosis complex}

Children with TSC and refractory partial epilepsy due to epileptogenic cortical tubers are potentially candidates for epilepsy surgery (47). If a primary epileptogenic tuber can be identified among several lesions through noninvasive studies or intracranial recording, focal surgical resection can have a favorable seizure outcome, comparable to results in extratemporal epilepsy for FCD (48).
General features of TSC include (Figure 9):

* Cortical and sub-cortical tubers which are frequently calcified, hyperintense in T2 sequences and hypointense in $\mathrm{T} 1$ sequences (viceversa in nonmyelinated infants brain), usually not enhancing;

* Subependymal hamartomas:

- Often presenting with calcification;

- Abnormal signal, frequently high T1 and iso to high T2;

- Enhancement is a variable feature, not useful in the differential diagnosis with subependymal giant cell astrocytomas (SGCA);

* SGCA. 
- Usually occur between 8 and 18 years;

- Frequently large and growing in time;

- Intense enhancement;

- Can cause obstruction of Monro foraminas and subsequent hydrocephalus;

- A lesion growing, and/or larger than $10 \mathrm{~mm}$ within the foramen of Monro is considered subependymal giant astrocytoma (SEGA) in a patient with TSC;

* White matter abnormalities:

- Usually nodular, ill-defined, cystic and bandlike lesions;

- "Radial bands".

\section{Polymicrogyria (PMG)}

Epilepsy associated with PMG has a large spectrum of electroclinical presentations as compared to other epileptic syndromes. Disappointing results on seizures following limited resection of the polymicrogyric malformation, as well as electrophysiological investigations of the apparently normal cortex surrounding focal PMG in an animal model, indicates that epileptogenesis is not limited to the visible cortical malformation (49).

General features of PMG:

Most frequently involved region is the perisylvian area ( $80 \%$ of cases), usually bilaterally ( $60 \%$ of cases).

* Frontal region: $70 \%$ of cases with sparing of gyrus rectus and cingulate gyrus;

- Parietal: 63\%;

* Temporal: $38 \%$ of cases with sparing of hippocampus;

- Occipital: 7\% of cases with sparing of visual cortex.

MRI represents the "gold standard" imaging technique in the evaluation of PMG, with thickening of the cortex being the most striking findings (usually in association with abnormal signal intensity). PMG is a probably a mechanism through which the brain compensates for damage during the foetal development when the brain is not able to develop gliosis and can be found in pre-natal infection (TORCH infections, Figure 10), after fetal vascular insults or can be genetically determined (e.g., Zelwegger syndrome, tubulinopathies, etc.) (50) (Figure 11).

\section{Signal intensity and morphology}

Cortical signal intensity in PMG is usually similar to normal grey matter. Due to the presence of dilated perivascular spaces (51), a hyperintense T2-w signal is frequently (20$27 \%$ ) recognizable in the adjacent subcortical white matter.
The numerous packed gyri ("lumpy bumpy" appearance) are really small and indeed usually visible only on thinsection high- resolution MRI (particularly 3D T1-WI); it's important to remember that this characteristic "appearance" may be subtle and therefore a differential diagnosis with pachygyria is often challenging, as both are associated with broad enlarged and often thickened gyri.

\section{Heterotopia}

The arrest of the physiological migration process of neuronal precursors along the radial pathway between the ependymal lining of the ventricles walls and the subcortical regions results in the presence of heterotopic grey matter. Heterotopia can be categorized into two big sub-groups: sub-ependymal and sub-cortical. The role of the nodular heterotopia in seizures development is complex but most likely they are involved together with the overlying cortex in the epileptogenic network. Imaging findings of heterotopic GM are mainly represented by the presence of non-enhancing nodules of grey matter intensity on all sequences (52).

\section{Hemimegalencephaly}

Hemimegalencephaly is a rare disease characterized by hamartomatous growth of one cerebral hemisphere or part of it and the key findings are the presence of an enlarged hemisphere with ipsilateral ventriculomegaly. The clinical presentation of these patients span from epileptic seizures and macrocrania to serious developmental delay with contralateral hemiparesis. Different patterns of structural abnormalities may be associated with cortical thickening, mainly represented by manifestations of the Lissencephaly-pachygyria spectrum and PMG. Later on, along with the progression of the disease, cerebral hemispheric atrophy may be seen due to continual epileptic activity. Anomalies in the mTOR pathway are associated with hemimegalencephaly (53).

\section{Schizencephaly}

Schizencephaly is a congenital cortical malformation characterized by an abnormal grey matter lined cleft, connecting the ventricular ependyma to the subarachnoid space. This pathology can be categorized in two main groups: open-lip schizencephaly in which the cleft walls are separated and closed-lip schizencephaly in which there is apposition between the walls. The cleft lining is usually constituted by polymicrogyric grey matter. It is frequently associated with other abnormalities, mainly represented by septo-optic dysplasia and grey matter heterotopia. 

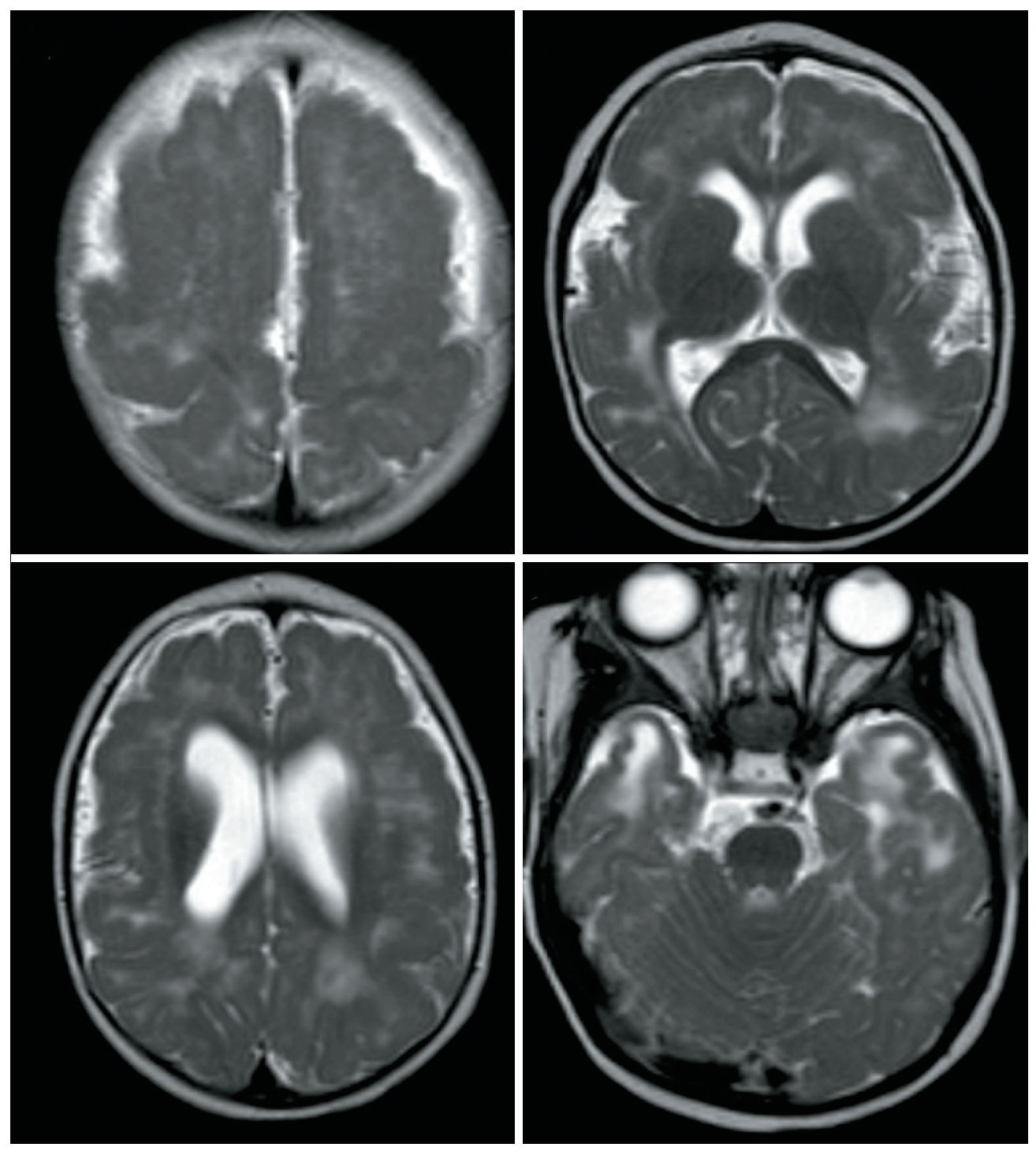

Figure 10 Axial T2-WI of an 8-month-old boy with upper and lower limbs spasticity, microcephaly and deafness showing the presence of diffuse PMG due to congenital CMV infection. T2-WI, T2-weighted images; PMG, polymicrogyria; CMV, cytomegalovirus.

\section{Neoplasms}

Among the most frequent causes of epileptic seizures in both pediatric and adult population, neoplasms represent a relative infrequent etiology (with incidence reported ranging from $2 \%$ to $4 \%$ ) (54). The most frequent area involved is the temporal lobe (68\% of cases), usually in a cortical/juxta-cortical location with no evident mass effect or surrounding edema, but often associated with calvarial remodeling (reflecting their chronic growth and indolent nature) (54). MRI is the "gold standard" technique for the detection of neoplasms, with a sensitivity of nearly $100 \%$.
Amid the diverse and heterogenous spectrum of neoplastic pathology causing epilepsy some histotypes are more frequent than others, such as low-grade astrocytic tumors, ganglioglioma, dysembryoplastic neuroepithelial tumor (DNET), pleomorphic xanthoastrocytoma, and cerebral metastasis. MRI plays an important role in helping radiologists to recognize some characteristic features important in the differential diagnosis process.

\section{Ganglioglioma}

Predominantly affecting patients under 30 years old, 

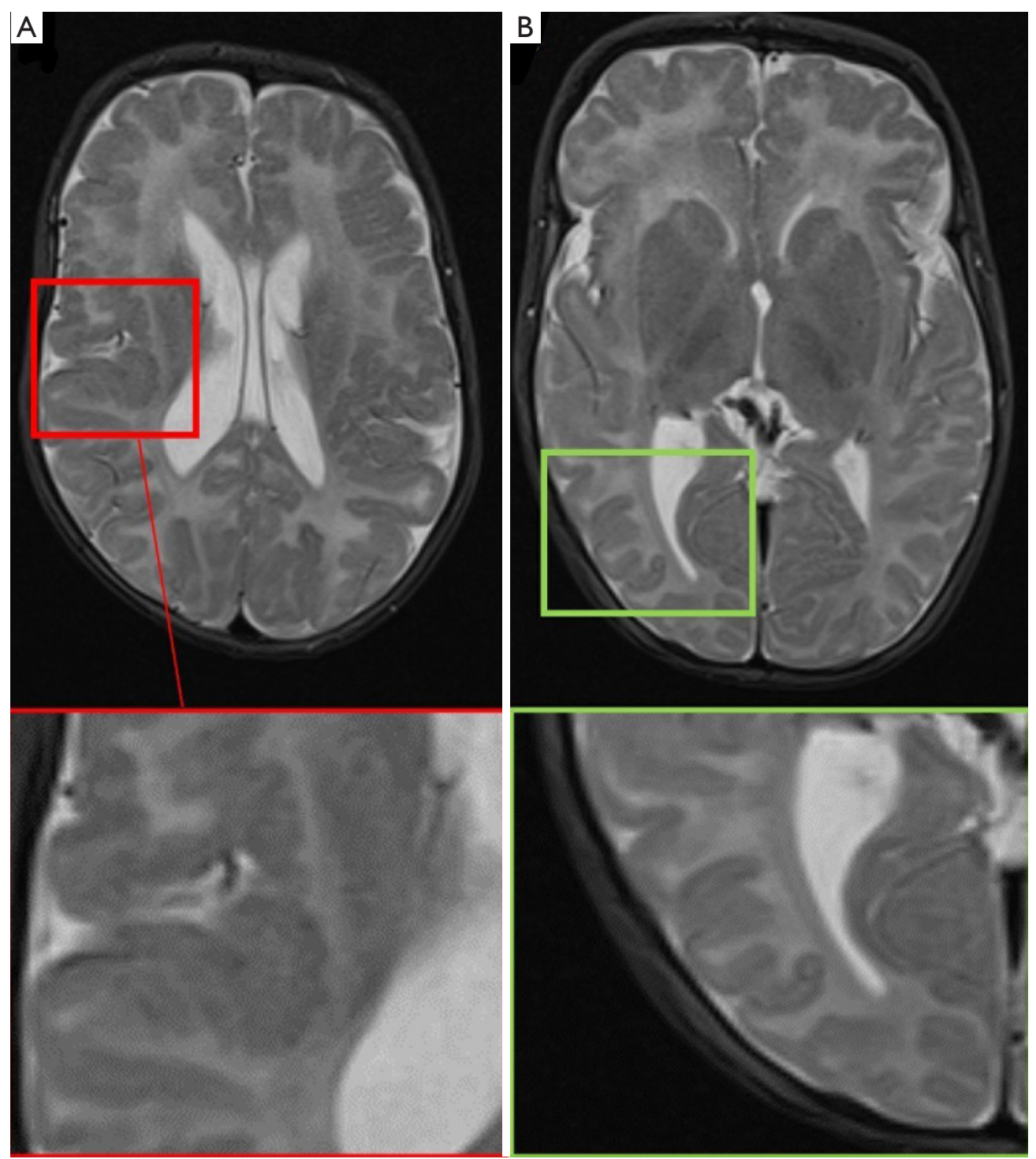

Figure 11 Axial T2-WI showing localized PMG in the right frontal (A) and temporo-parietal areas (B), in a 2-week-old boy with seizures and hypotonia with confirmed Zellweger syndrome (PEX1 gene). T2-WI, T2-weighted images; PMG, polymicrogyria.

ganglioglioma is a benign, indolent neoplasm which is frequently cortically based and most commonly located in temporal lobes. Usually presenting as a mixed solid-cystic lesion characterized by the presence of calcifications, it shows only minimal or no mass effect/peri-lesional edema. After administration of paramagnetic contrast agent it shows a variable and often dishomogeneous enhancement (54) (Figure 12).

\section{Dysembryoplastic neuroepithelial tumors}

DNETs are indolent neoplasms typically encountered in the pediatric population, usually cortically-based, appearing as dishomogeneous lesions (cystic-nodular features); MRI typically shows a multicystic lesion ("bubbly" appearance on T2-w sequences) characterized by a mixed signal intensity on FLAIR (often showing "bright rim" sign), with subtle or no enhancement at all (Figure 13).

\section{Pleomorphic xanthoastrocytomas}

Pleomorphic xanthoastrocytomas have the typical appearance of the low-grade astrocytomas in children, characterized by a mixed solid-cystic lesion with an enhancing mural nodule. A typical feature of this particular 

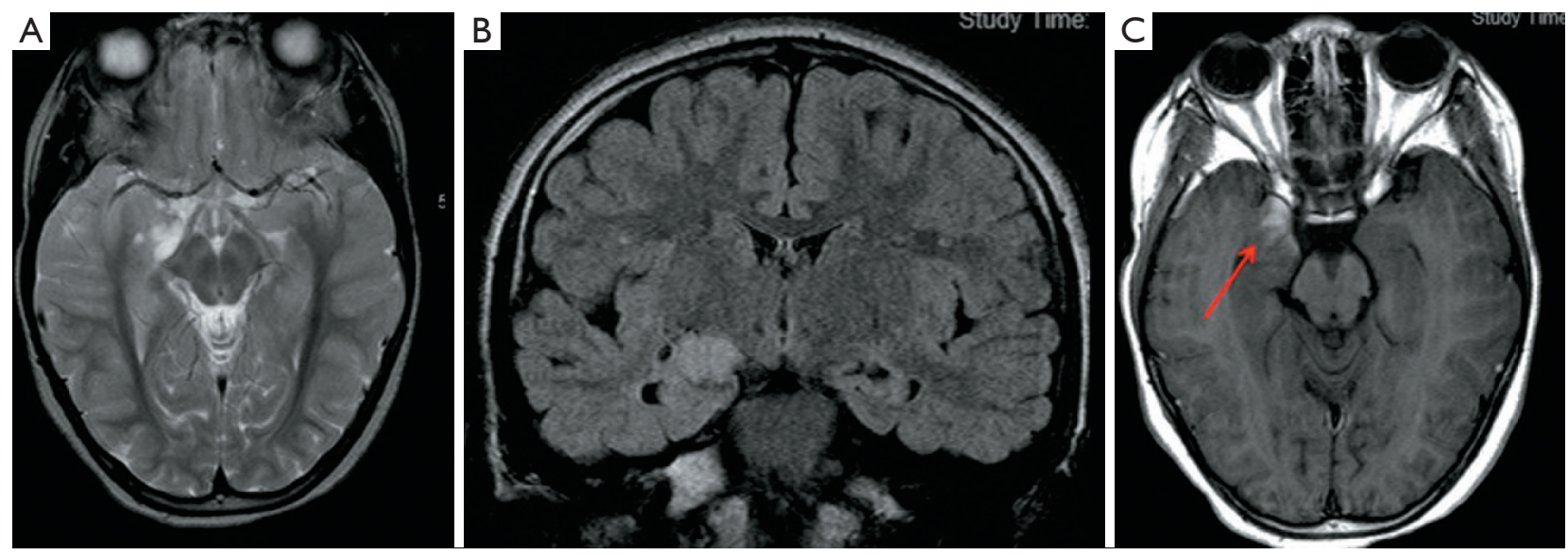

Figure 12 Axial T2-w (A), coronal FLAIR (B) and axial T1-w (C) images showing a right temporal pole enhancing lesion (red arrow) compatible with ganglioglioma in a 7-year-old female with temporal lobe seizures. T2-w, T2-weighted; FLAIR, fluid-attenuated inversion recovery sequence; T21-w, T1-weighted.

subgroup of astrocytomas is the involvement of the adjacent leptomeninges, which appear thickened and enhancing after administration of contrast agent. Even if surgical approach is characterized by good prognosis, there is a variable rate of loco-regional recurrence and/or malignant transformation (55).

Multinodular and vacuolating neuronal tumor (MVNT) MVNT is a benign neuronal tumor that is newly recognized as architectural appearance that may be related to ganglion cell tumors in 2016 World Health Organization Classification of Tumors of the Central Nervous System. They are characterized by multiple tumor nodules, vacuolar alteration, and widespread immunolabeling for human neuronal protein $\mathrm{HuC} / \mathrm{HuD}$. These tumors appear as a cluster of well-circumscribed high T2 signal 'bubbles' located predominantly in the subcortical white matter but can involve the overlying cortex (56).

\section{Hypothalamic hamartoma}

Hypothalamic hamartoma is a benign indolent lesion, also known as diencephalic or tuber cinereum hamartoma. It represents non-neoplastic congenital grey matter heterotopia in the region of tuber cinereum of the hypothalamus. It is seen in infants presenting with epilepsy, usually characterized by gelastic seizures, and precocious puberty.

\section{Vascular malformations}

Vascular malformations represent a less frequent etiology of focal epilepsy in children; they can be broadly categorized into two main subgroups:

* Arteriovenous malformations (AVMs);

* Cavernous malformations.

AVMs is the most common in the pediatric population compared to cavernous malformations, are usually congenital and basically consist of a tangle of anomalous blood vessels which lead to a direct blood arteriovenous shunting (pathologically lacking the normal "capillary network") and can be affected by secondary changes such as thrombosis, calcification and hemorrhage. MRI findings are represented by serpiginous flow voids with areas of $\mathrm{T} 2$ prolongation in the adjacent brain parenchyma in T1-WI and T2-WI. Cavernous malformations are basically composed of well circumscribed vascular spaces containing blood in various stages of evolution. These lesions can be numerous and $50 \%$ to $80 \%$ of multiple lesions occur on a familial basis. Characteristic imaging findings are a "popcorn like" lesion marked by a heterogeneous central hyperintensity on all pulse sequences, with a surrounding "rim" of low signal due to the presence of hemosiderin. Because hemosiderin results in magnetic susceptibility artifacts (which are visualized as signal voids on MR images), sequences that are more affected by magnetic susceptibility artifacts will tend to have the greatest sensitivity for detecting small cavernomas $(57,58)$. These sequences are particularly important in case of familial multiple cavernous syndrome (Figure 14) due to specific genes such as KRIT1, CCM2 and PDCD10 and in those cases a spine MRI should also be performed $(59,60)$. A short mention should be done about cerebral 

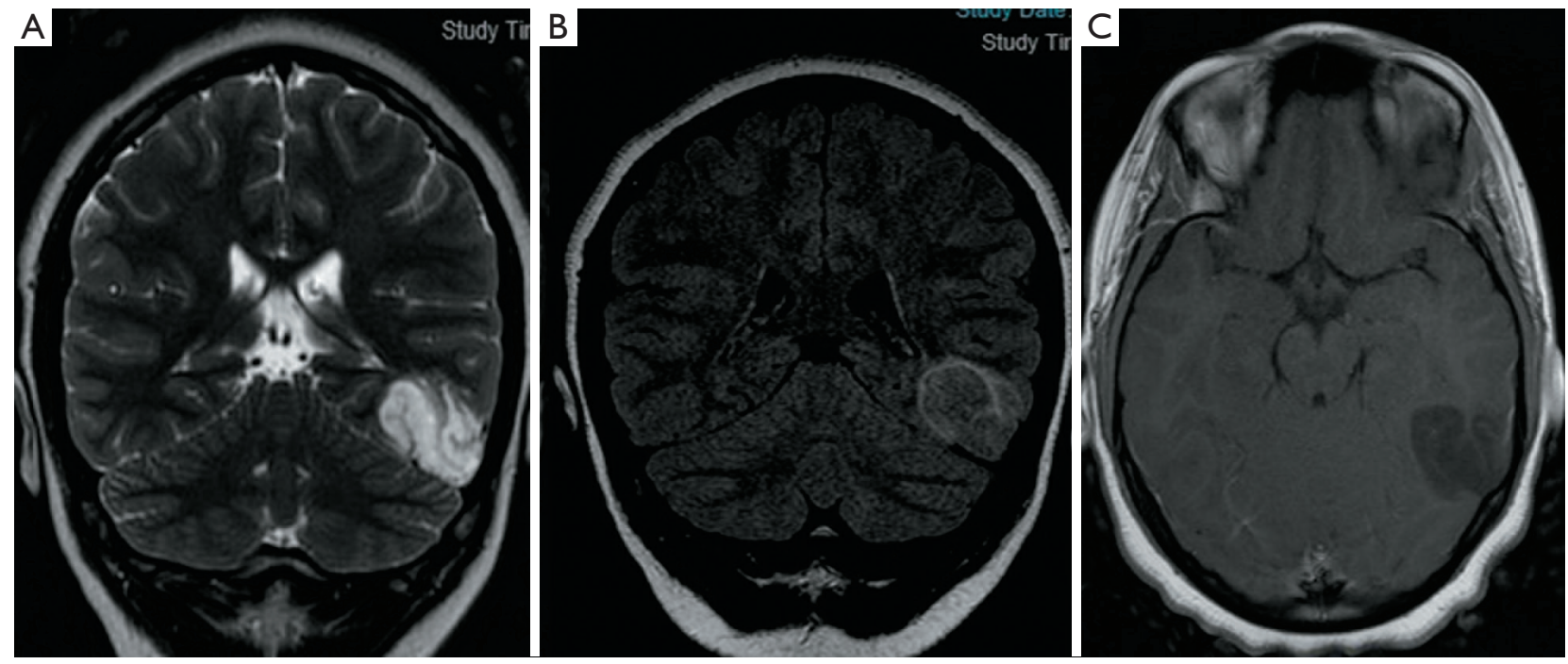

Figure 13 Coronal T2-w (A), coronal FLAIR (B) and axial T1-w post-contrast (C) images showing a left temporal lobe non-enhancing lesion with an hyperintense rim on FLAIR and a "bubbly" appearance on T2, compatible with DNET. T2-w, T2-weighted; FLAIR, fluidattenuated inversion recovery sequence; T1-w, T1-weighted; DNET, dysembryoplastic neuroepithelial tumor.

venous angioma, a congenital malformation of veins which drain normal brain, very common as incidental finding, most commonly located in the fronto-parietal region and in cerebellar hemispheres. Developmental venous anomalies are often visible on most sequences but can be subtle and are most easily seen on post-contrast $\mathrm{T} 1$ sequences and susceptibility weighted imaging (SWI).

\section{Prenatal, perinatal, and postnatal insults}

The timing of clastic/destructive pre-, peri- or post-natal insults is crucial in defining the appearance and the imaging findings of these lesions; Early damage to the fetus brain can lead to either MCD (e.g., PMG) or, later on in the fetal development, to the formation of smooth walled cavities with absent glial reaction in the surrounding parenchyma, a process known as "porencephaly". These porencephalic cavities are usually seen in frontal-perisylvian regions, are filled with CSF and may communicate with the ventricles and/or with subarachnoid space. Porencephaly is typically related with increased incidence of mesiotemporal sclerosis both unilaterally or bilaterally. If the insult occurs during the peri- or postnatal interval it may lead to a characteristic pattern of encephalomalacia or ulegyria. Encephalomalacia may result from late gestational, perinatal, and postnatal injuries to the brain (at this time the infant brain has gained the ability to develop gliosis). Encephalomalacia results in multiple, irregular cystic cavities with prominent astrocytic proliferation. Ulegyria, usually due to global hypoxicischemic injury in term infants, is usually located in the parieto-occipital region. It is characterized by destruction of the grey matter in the depths of the sulci, sparing the crowns of gyral convolutions.

\section{Post-traumatic epilepsy}

Post-traumatic brain damage represents one of the etiologies of epilepsy, especially in the neonatal patients. The most common locations of brain injury in trauma are along the antero-inferior regions of the brain, due to the presence of bony skull irregularities at these locations (especially in the frontal and temporal poles) (61). These post-traumatic shearing injuries and contusions are usually associated with hemorrhage, resulting in hemosiderin deposition and reparative gliotic changes. Hemosiderin deposits and gliotic changes are known to be involved in seizure generation/ propagation and there is evidence of a $25 \%$ risk of developing post-traumatic epilepsy in patients with intraparenchymal or extra-axial hemorrhage and depressed skull fractures (62). MR (with SWI sequences) represents a useful tool to assess the presence of diffuse axonal injury (DAI), intracerebral hematoma, subdural hemorrhage, contusions and gliosis.

\section{Rasmussen's encephalitis}

Rasmussen's encephalitis is a pediatric disease, in which children typically present with epilepsy and developing 


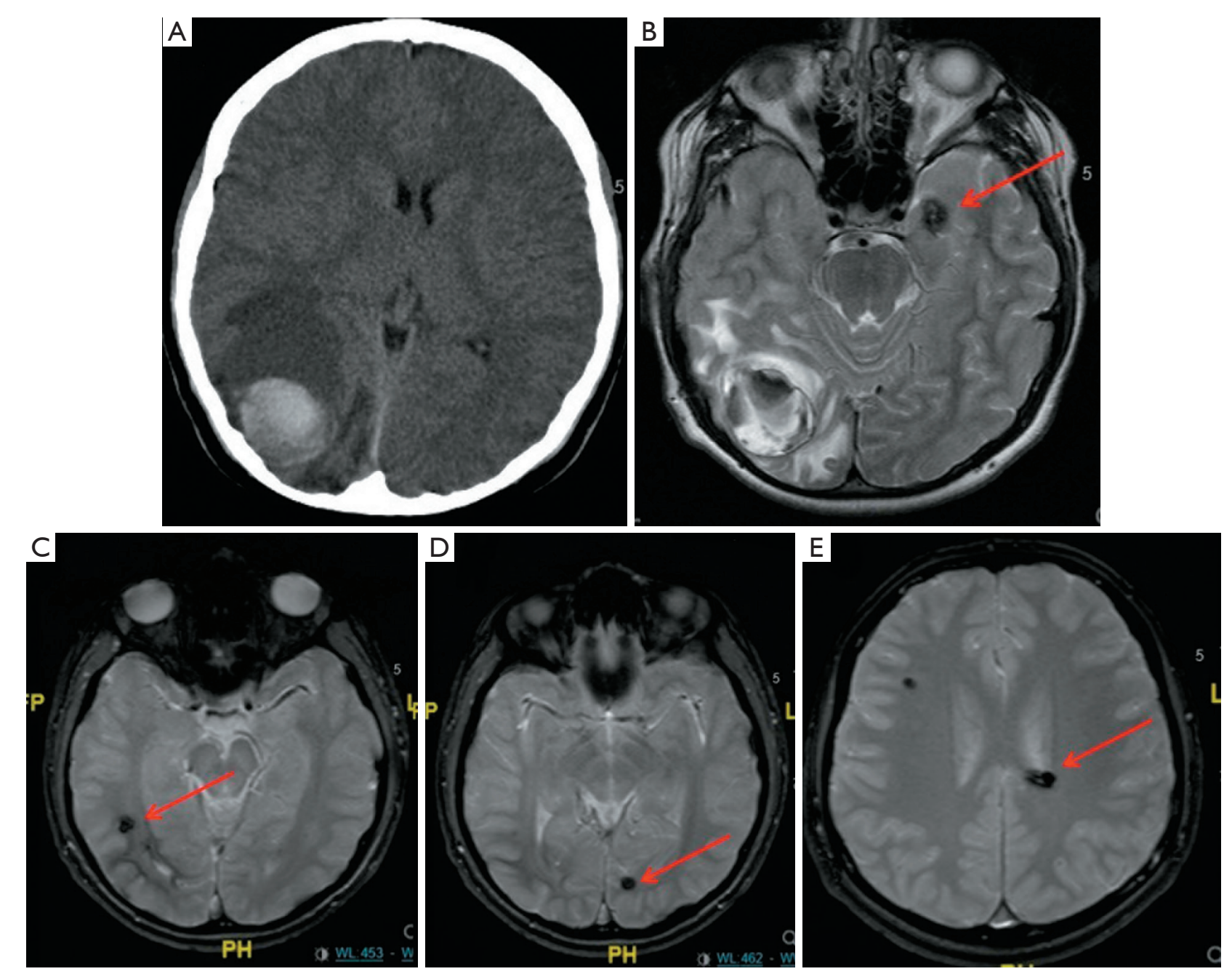

Figure 14 Axial CT (A) and axial T2* GE (B-E) images performed in a 14-year-old male with sudden onset of seizures, vomiting and lethargy. Images show a large intra-axial hematoma in the right parietal lobe and multiple hypointense lesions on T2* sequences ("blooming effect”) in the cerebral hemispheres (red arrows) compatible with cavernous malformations (familiar cerebral cavernous malformation).

neurologic deficits and cognitive impairment. Usually affecting only one hemisphere, which slowly becomes atrophic during the course of the disease (with associated enlargement of the ventricles), it appears on MR as an area of T2 signal prolongation in the cortex and subcortical WM (predominantly involving the fronto-insular region). Usually no CT or MRI abnormalities are seen in the early phase of the disease, then some hyperintense signal alterations on T2-w sequences are recognizable typically involving the white matter and putamen.

\section{Sturge-Weber syndrome}

MRI is the modality of choice for the assessment of SWS that is a phakomatosis characterized by deranged vasculature of the face (ipsilateral facial angioma in the distribution of the trigeminal nerve), brain and meninges (angiomatosis of the leptomeninges). Poor venous drainage and chronic ischemic damage to the underlying cortex develop as a result of the leptomeningeal vascular malformation. Clinically these patients present with multiple and intractable epileptic seizures. MR usually shows the structural abnormalities in the brain, including pial angiomata in the parietal occipital region on post-contrast-enhanced images, cortical calcifications subjacent to the cortex and white matter in the parietooccipital region, enlarged choroid plexus and atrophy of the ipsilateral cerebral hemisphere (same side of the angioma). 


\section{Pre-surgical planning}

Qualitative evaluation of epileptic substrate on MRI represents a fundamental step in the process of pre-surgical assessment of epileptic patients, implemented with clinical and EEG information. The closeness to eloquent cortex, optic radiation and corticospinal tract represents an important element in the pre-surgical decisional process to help optimize the surgical approach and minimize the surgical risks (63) and can be assessed using additional quantitative techniques such as fMRI, DTI. If no relevant lesions are found on MRI, further acquisitions using the newest MRI hardware and techniques and post-acquisition processing methods might help revealing subtle abnormalities, but findings should be interpreted with caution owing to the possibility of false positive results. 18F-fluorodeoxyglucose (18F-FDG) positron emission tomography (PET) represents an important additional step because of its utility to identify areas of hypometabolism which may indicate a possible epileptogenic zone. If no abnormalities are recognizable on 18F-FDG PET imaging, subsequent additional investigations are usually requested. These methods include ictal single photon emission computed tomography (SPECT) and visualization of interictal epileptic activity with electrical source imaging (ESI), SEEG and simultaneous EEG and fMRI (EEG-fMRI) (64).

\section{Mapping eloquent brain functions (MEF)}

In the multimodality approach to epileptic patients an important role is played by functional MR techniques, fundamental to assess the speech lateralization and to evaluate eloquent areas of the cortex. This process becomes even more important in the pre-surgical planning of patients with epileptic foci located nearby eloquent areas of the brain.

\section{Language}

fMRI is broadly used to map cortical responses to different speech inputs, highlighting cortical activations (either in Broca's area or Wernicke's) associated with different types of speech stimuli and/or tasks performed by the patient. This process helps to assess the different patterns of typical and atypical language lateralization (65). Most recently some authors (66) have studied the role of fMRI in the discrimination of different individual speech sounds, such as individual vowels, syllables, words or concepts.

\section{Motor function}

fMRI with finger and foot tapping tasks can be used to identify the primary motor cortex, that is a crucial step when planning intracranial EEG implantations and resections (67) (Figure 15).

\section{Mapping cerebral white matter connections}

Another important thing that should be taken in account when planning epileptic surgery is avoiding surgical damage to white matter connections to prevent post-surgical neurological deficits. Tractography data derived from diffusion-weighted MRI, usually DTI, enables the noninvasive in-vivo delineation of white matter tracts. Most clinical research into white matter tracts in patients with epilepsy has focused on the optic radiation because damage to Meyer's loop during anterior temporal lobe resection can cause a important visual field deficit that can preclude many everyday activities (68). The extent of resection and distance from Meyer's loop to the temporal pole on preoperative tractography are predictive of the risk of a visual field deficit (69), and tractography therefore can be used to assist with surgical planning and risk stratification (70). Another example of the crucial role of tractography in the pre-surgical assessment of epileptic patients, especially in children, is represented by the evaluation of the cortico-spinal tracts in patients undergoing frontal lobes surgery; this tool has shown to be very useful in predicting the potential risks of postoperative motor deficits (71) (Figure 16).

\section{Localization of epileptic activity}

Additional investigations other than MRI are often necessary to assess the exact localization of the epileptic foci, especially when there is no concordance between imaging findings and clinical/EEG data. A non-invasive investigation technique useful for the localization of epileptogenic brain areas, especially in MRI-negative focal epilepsies, is represented by $18 \mathrm{~F}-\mathrm{FDG}-\mathrm{PET}$ imaging which has been used for years in the assessment of surgical epilepsies before the advent of MRI. PET has been used for many years to help neuroradiologists and clinicians in the localization of epileptic foci. It represents a versatile and feasible method capable of providing dynamic information regarding local and general metabolism in the brain. Glucose metabolism is the most commonly measured parameter using 18F-FDG. The usual finding in epileptic patients is represented by a regional reduction in glucose uptake (hypometabolism) during the interictal state. A significant increase during ictal seizure activity has also been reported. There seems to be a significant relation between the time of the last seizure and the degree 
of observed regional hypometabolism in epilepsy patients. The exact reasons for the interictal hypometabolism are still unclear. A common clinical presumption suggests that cerebral hypometabolism reflects neuronal cell loss, however, in epilepsy patients hypometabolism more closely reflects the degree of cerebral dysfunction than it does actual structural compromise, part of which may be due to loss of synaptic inputs, in addition to the electrical activity generated within the dysfunctional cortex (72). Usually PET imaging is preferred to ictal SPECT (see later) in the epilepsy pre-surgical approach, due to its wide availability and its potential use as an interictal investigation. Regional cerebral hypometabolism recognizable with $18 \mathrm{~F}-\mathrm{FDG}$ PET has often a wider distribution than that of the seizure

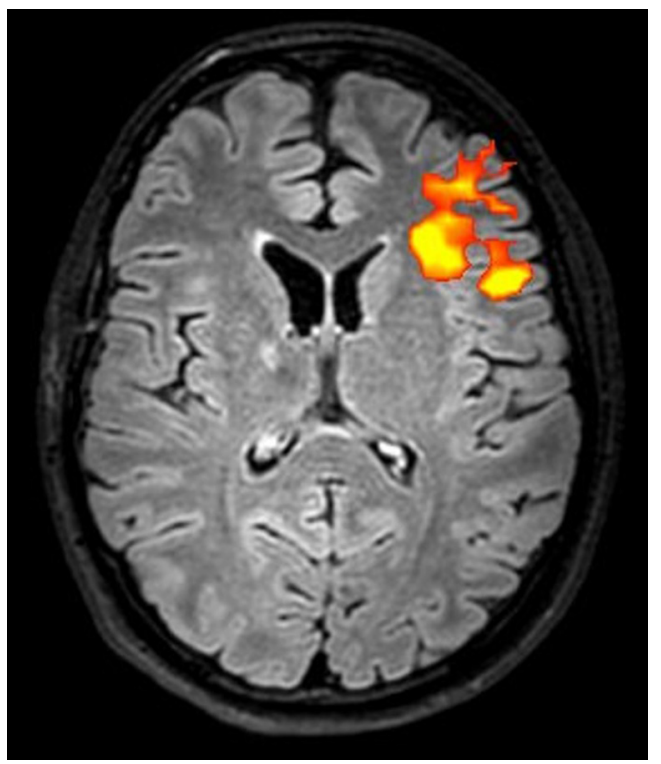

Figure 15 Speech fMRI of an epileptic patient showing cortical activation in Broca's area. fMRI, functional MRI. focus (73) (Figure 17). SPECT imaging can be really useful providing clues about the dynamic changes pre-, during and post-epileptic seizures and due to its dynamic nature the timing of injection is critical for the success of the exam and for the interpretation of the images. Hemodynamic changes seen on SPECT and occurring during the ictal phase are mainly represented by hyperperfusion in the epileptogenic region, usually surrounded by an area of hypo-perfusion that might be caused by shifting of blood flow to the seizure focus or might represent an inhibitory zone that limits the seizure spread (74). The simultaneous recording of scalp EEG while performing $\mathrm{fMRI}$ sequences represents a relative recent technique that can be used to evaluate the hemodynamic changes happening during the inter-ictal epileptic discharges, with a sensitivity ranging from $30 \%$ to $40 \%$ (75), and can be used as a tool to plan intracranial implantation (76), with widespread abnormalities taken as a warning sign of poor outcome from resection (77). A simultaneous ictal EEGfMRI recording in a patient presenting with frequent seizures can show focal or extensive hemodynamic changes on the fMRI images, typically starting before the onset of seizures on scalp EEG, suggesting that multiple additional brain networks might be involved in the epileptic phenomena (78) and highlighting the low sensitivity of scalp EEG (79). One example of the complementary role of both scalp EEG-fMRI is represented by the evidence (80) of cortico-subcortical pathways activation (seen on fMRI) during generalized spike wave discharges. Another important application of scalp EEG-fMRI in clinical practice is the possibility to recognize the different ictal and interictal brain networks in a pre-surgical assessment setting, helping the clinicians in stratifying the surgical risks for each different patient.

\section{Magnetoencephalography (MEG)}

MEG is a technique that helps localize sources of electrical

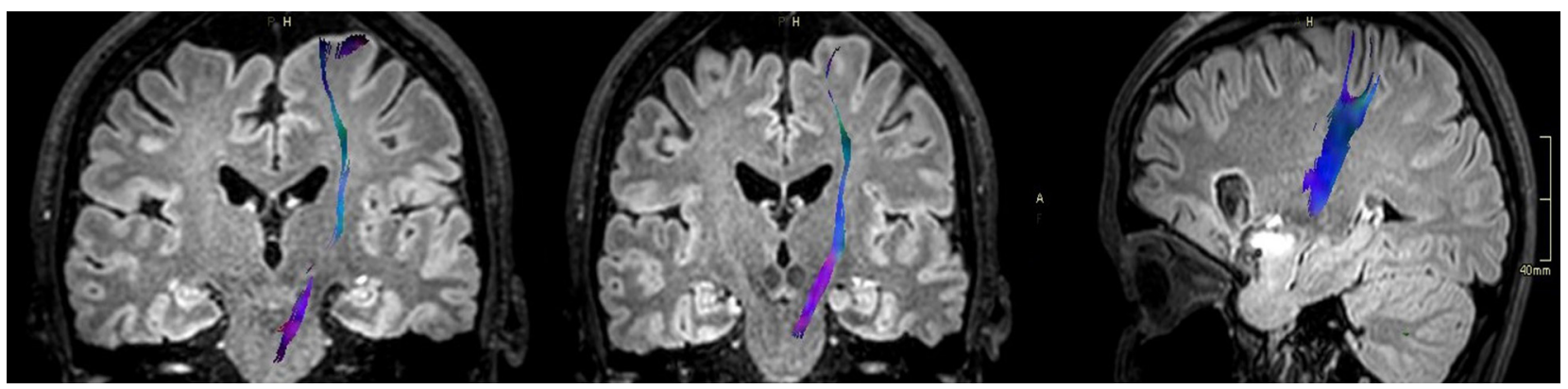

Figure 16 DTI tractography the corticospinal tract in an epileptic patient as pre-surgical assessment. DTI, diffusion tensor imaging. 

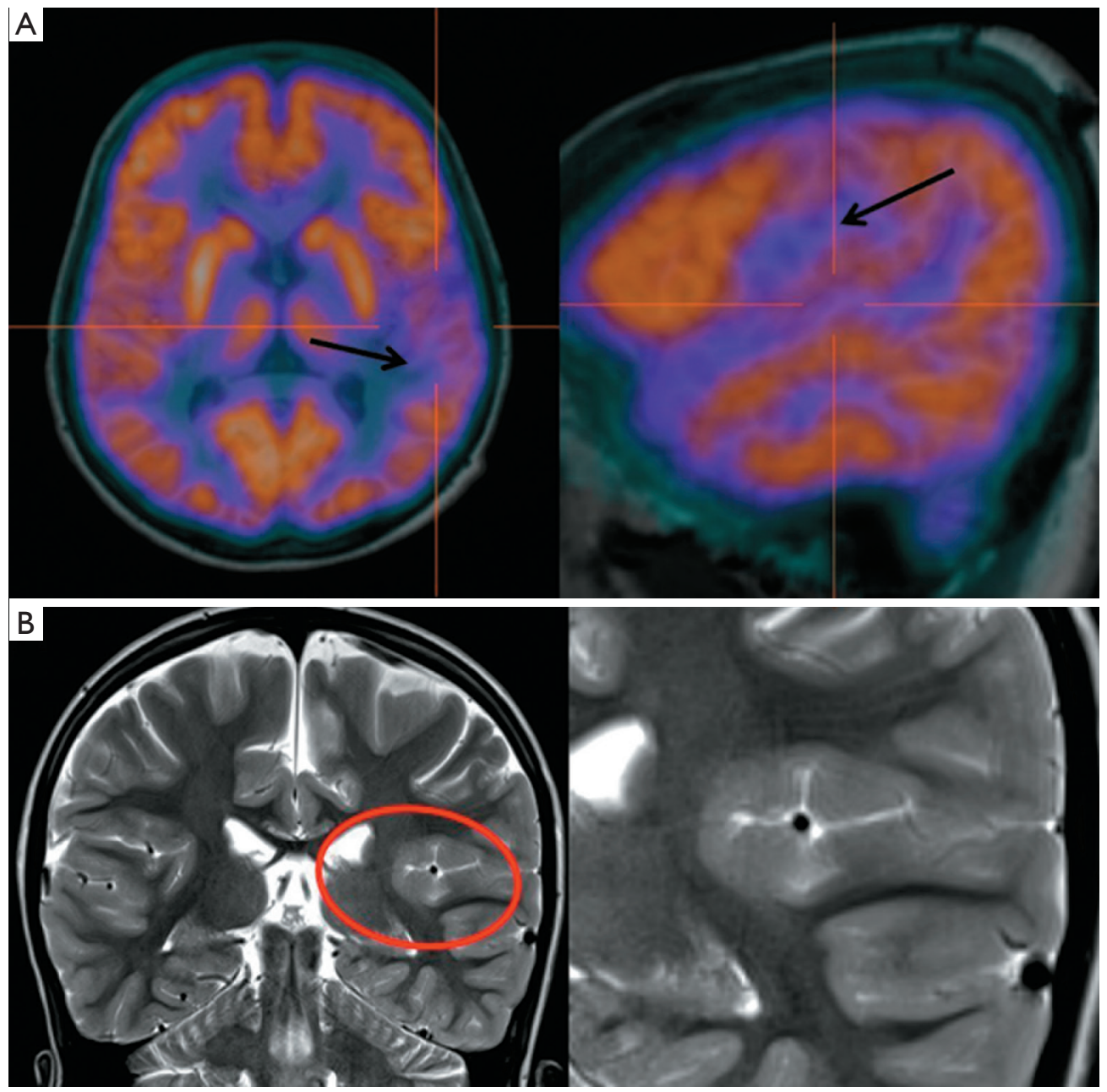

Figure 17 Inter-ictal PET (A) of an 8-year-old male with focal seizures showing a large area of hypometabolism in the left fronto-insular region. T2-w coronal images (B) showing the presence of FCD in the corresponding area (red circle). PET, positron emission tomography; T2-w, T2-weighted; FCD, focal cortical dysplasia.

activity within the human brain by non-invasively measuring the magnetic fields arising from such activity. Brain neuronal activity generates electrical currents, which in turn generate electrical field potentials detectable by the EEG. These neuronal currents also produce a magnetic field that is detectable by MEG. However, while the EEG measures extra cellular currents, the MEG is a measure of the intracellular currents generated by the apical dendrites. One of the major advantages of this technique over the EEG is the lack of distortion of MEG signals by the skull and intervening soft tissue. In addition, the MEG preferentially records activity from tangential sources thus recording activity predominantly from sulci, which is not contaminated by activity from apical gyral (radial) sources.
While the MEG is probably more sensitive than the EEG in detecting interictal spikes, especially in some locations such as the superficial frontal cortex and the lateral temporal neocortex, both techniques are usually complementary to each other (81).

\section{Stereo-electroencephalography (SEEG)}

Intracranial EEG is a fundamental tool in defining the epileptogenic zone (82) in patients undergoing surgery and, together with the stereotactic placement of several depth electrodes, is known as SEEG. The electrodes are able to record from the area (within $1 \mathrm{~cm}$ radius) around the cerebral entry point to the distal end (target). To minimize the potential risk of complications (such as hemorrhage 
and infection) (83), a careful pre-operative planning of the electrode implantation must be obtained using multimodal imaging techniques. Precise planning can also improve the efficiency of the recording by ensuring that electrode contacts sample grey rather than white matter. The efficacy of SEEG can be difficult to assess. The accuracy of SEEG in localizing the $\mathrm{EZ}$ will depend primarily on the accuracy of the preimplantation hypothesis, as well as the adequacy of electrode coverage and interpretation of the recordings and any stimulation testing. Different centers and patients may have varying criteria and thresholds for when they will pursue an implant and when the findings are believed to justify surgical treatment. Finally, outcomes from any treatment interventions that follow will depend heavily on the focality and operability of the underlying epilepsies chosen for investigation and the options and choice of surgical treatment technique. In a recent meta-analysis of 33 studies including 2,959 patients who underwent SEEG, the $\mathrm{EZ}$ was identified in $92 \%$ of the patients, $72 \%$ were eligible for epilepsy surgery, and $33 \%$ were seizure free after surgery (84).

\section{Summary}

Imaging children with epilepsy poses specific challenges and a multidisciplinary approach involving electrophysiologists, neurologists, nuclear medicine experts, radiologists and clinical psychologists is mandatory. Knowing the technical differences between a standard pediatric MRI for cases with epilepsy is critical for the radiologist as well as being familiar with the different pattern and pathologies responsible for seizures in children.

\section{Acknowledgments}

Funding: None.

\section{Footnote}

Provenance and Peer Review: This article was commissioned by the editorial office, Translational Pediatrics for the series "Pediatric Neuroradiology for Trainees and Fellows: An Updated Practical Guide". The article has undergone external peer review.

Reporting Checklist: The authors have completed the Narrative Review reporting checklist. Available at http:// dx.doi.org/10.21037/tp-20-261
Peer Review File: Available at http://dx.doi.org/10.21037/ tp-20-261

Conflicts of Interest: All authors have completed the ICMJE uniform disclosure form (available at http:// dx.doi.org/10.21037/tp-20-261). The series "Pediatric Neuroradiology for Trainees and Fellows: An Updated Practical Guide" was commissioned by the editorial office without any funding or sponsorship. FD served as the unpaid Guest Editor of the series and serves as an unpaid editorial board member of Translational Pediatrics from Sep 2019 to Oct 2021. AC reports grants from Great Ormond Street Children's Charity, during the conduct of the study. The other authors have no other conflicts of interest to declare.

Ethical Statement: The authors are accountable for all aspects of the work in ensuring that questions related to the accuracy or integrity of any part of the work are appropriately investigated and resolved.

Open Access Statement: This is an Open Access article distributed in accordance with the Creative Commons Attribution-NonCommercial-NoDerivs 4.0 International License (CC BY-NC-ND 4.0), which permits the noncommercial replication and distribution of the article with the strict proviso that no changes or edits are made and the original work is properly cited (including links to both the formal publication through the relevant DOI and the license). See: https://creativecommons.org/licenses/by-nc-nd/4.0/.

\section{References}

1. Proposal for revised classification of epilepsies and epileptic syndromes. Commission on Classification and Terminology of the International League Against Epilepsy. Epilepsia 1989;30:389-99.

2. Hauser WA. The prevalence and incidence of convulsive disorders in children. Epilepsia 1994;35 Suppl 2:S1-6.

3. Begley CE, Famulari M, Annegers JF, et al. The cost of epilepsy in the United States: an estimate from populationbased clinical and survey data. Epilepsia 2000;41:342-51.

4. Kwan P, Brodie MJ. Early identification of refractory epilepsy. N Engl J Med 2000;342:314-9.

5. Blume WT, Lüders HO, Mizrahi E, et al. Glossary of descriptive terminology for ictal semiology: report of the ILAE task force on classification and terminology. Epilepsia 2001;42:1212-8. 
6. Blumenfeld H. Impaired consciousness in epilepsy. Lancet Neurol 2012;11:814-26.

7. Coryell J, Gaillard WD, Shellhaas RA, et al. Neuroimaging of early life epilepsy. Pediatrics 2018;142:e20180672.

8. Baumgartner C, Koren JP, Britto-Arias M, et al. Presurgical epilepsy evaluation and epilepsy surgery. F1000Res 2019;8.1818.

9. Hakami T, McIntosh A, Todaro M, et al. MRI-identified pathology in adults with new-onset seizures. Neurology 2013;81:920-7.

10. Wilmshurst JM, Gaillard WD, Vinayan KP, et al. Summary of recommendations for the management of infantile seizures: Task Force Report for the ILAE Commission of Pediatrics. Epilepsia 2015;56:1185-97.

11. Panayiotopoulos CP. Benign childhood focal seizures and related epileptic syndromes. In: Panayiotopoulos CP. A clinical guide to epileptic syndromes and their treatment. London: Springer, 2010:339-75.

12. Rastogi S, Lee C, Salamon N. Neuroimaging in pediatric epilepsy: a multimodality approach. Radiographics 2008;28:1079-95.

13. Fitsiori A, Hiremath SB, Boto J, et al. Morphological and advanced imaging of epilepsy: beyond the basics. Children (Basel) 2019;6:43.

14. Zijlmans M, de Kort GAP, Witkamp TD, et al. 3T versus $1.5 \mathrm{~T}$ phased-array MRI in the presurgical work-up of patients with partial epilepsy of uncertain focus. J Magn Reson Imaging 2009;30:256-62.

15. Martinez-Rios C, McAndrews MP, Logan W, et al. MRI in the evaluation of localization-related epilepsy. J Magn Reson Imaging 2016;44:12-22.

16. Knake S, Triantafyllou C, Wald LL, et al. 3T phased array MRI improves the presurgical evaluation in focal epilepsies: a prospective study. Neurology 2005;65:1026-31.

17. Mellerio C, Labeyrie MA, Chassoux F, et al. 3T MRI improves the detection of transmantle sign in type 2 focal cortical dysplasia. Epilepsia 2014;55:117-22.

18. Rubinger L, Chan C, D'Arco F, et al. Change in presurgical diagnostic imaging evaluation affects subsequent pediatric epilepsy surgery outcome. Epilepsia 2016;57:32-40.

19. Blair RD. Temporal lobe epilepsy semiology. Epilepsy Res Treat 2012;2012:751510.

20. Von Oertzen J, Urbach H, Jungbluth S, et al. Standard magnetic resonance imaging is inadequate for patients with refractory focal epilepsy. J Neurol Neurosurg Psychiatry 2002;73:643-7.

21. Adler S, Hong SJ, Liu M, et al. Topographic principles of cortical fluid-attenuated inversion recovery signal in temporal lobe epilepsy. Epilepsia 2018;59:627-35.

22. Bernasconi A, Cendes F, Theodore WH, et al. Recommendations for the use of structural magnetic resonance imaging in the care of patients with epilepsy: a consensus report from the International League Against Epilepsy Neuroimaging Task Force. Epilepsia 2019;60:1054-68.

23. Lee YJ. Advanced neuroimaging techniques for evaluating pediatric epilepsy. Clin Exp Pediatr 2020;63:88-95.

24. Zhang Q, Eagleson R, Peters TM. Volume visualization: a technical overview with a focus on medical applications. J Digit Imaging 2011;24:640-64.

25. Hildebrand MS, Harvey AS, Malone S, et al. Somatic GNAQ mutation in the forme fruste of Sturge-Weber syndrome. Neurol Genet 2018;4:e236.

26. Grade M, Hernandez Tamames JA, Pizzini FB, et al. A neuroradiologist's guide to arterial spin labeling MRI in clinical practice. Neuroradiology 2015;57:1181-202.

27. Daghistani R, Widjaja E. Role of MRI in patient selection for surgical treatment of intractable epilepsy in infancy. Brain Dev 2013;35:697-705.

28. Bronen RA, Fulbright RK, Spencer DD, et al. MR characteristics of neoplasms and vascular malformations associated with epilepsy. Magn Reson Imaging 1995;13:1153-62.

29. Thom M. Review: hippocampal sclerosis in epilepsy: a neuropathology review. Neuropathol Appl Neurobiol 2014;40:520-43.

30. Siegler Z, Barsi P, Neuwirth M, et al. Hippocampal sclerosis in severe myoclonic epilepsy in infancy: a retrospective MRI study. Epilepsia 2005;46:704-8.

31. Dunleavy M, Shinoda S, Schindler C, et al. Experimental neonatal status epilepticus and the development of temporal lobe epilepsy with unilateral hippocampal sclerosis. Am J Pathol 2010;176:330-42.

32. Hsu CCT, Stenberg L, Krings T. Imaging the patient with epilepsy. In: Hodler J, Kubik-Huch RA, von Schulthess GK. editors. Diseases of the brain, head and neck, spine 2020-2023: diagnostic imaging. Cham: Springer, 2020.

33. Chan S, Erickson JK, Yoon SS. Limbic system abnormalities associated with mesial temporal sclerosis: a model of chronic cerebral changes due to seizures. Radiographics 1997;17:1095-110.

34. Coan AC, Kobayashi E, Lopes-Cendes I, et al. Abnormalities of hippocampal signal intensity in patients with familial mesial temporal lobe epilepsy. Braz J Med Biol Res 2004;37:827-32. 
35. Cheon JE, Chang KH, Kim HD, et al. MR of hippocampal sclerosis: comparison of qualitative and quantitative assessments. AJNR Am J Neuroradiol 1998;19:465-8.

36. Bernasconi A, Bernasconi N, Caramanos Z, et al. T2 relaxometry can lateralize mesial temporal lobe epilepsy in patients with normal MRI. Neuroimage 2000;12:739-46.

37. Tsai MH, Vaughan D, Perchyonok Y, et al. Hippocampal malrotation is an anatomic variant and has no clinical significance in MRI-negative temporal lobe epilepsy. Epilepsia 2016;57:1719-28.

38. Sirven JI. Epilepsy: a spectrum disorder. Cold Spring Harb Perspect Med 2015;5:a022848.

39. Pang T, Atefy R, Sheen V. Malformations of cortical development. Neurologist 2008;14:181-91.

40. Alami B, Tazi L, Traoré Z, et al. Magnetic resonance imaging findings and Spectrum of Etiologies in children epilepsy. European Congress of Radiology 2015. doi: 10.1594/ecr2015/C-2262.

41. Colliot O, Bernasconi N, Khalili N, et al. Individual voxelbased analysis of gray matter in focal cortical dysplasia. Neuroimage 2006;29:162-71.

42. Leventer RJ, Guerrini R, Dobyns WB. Malformations of cortical development and epilepsy. Dialogues Clin Neurosci 2008;10:47-62.

43. Kabat J, Król P. Focal cortical dysplasia - review. Pol J Radiol 2012;77:35-43.

44. Blümcke I, Thom M, Aronica E, et al. The clinicopathologic spectrum of focal cortical dysplasias: a consensus classification proposed by an ad hoc Task Force of the ILAE Diagnostic Methods Commission. Epilepsia 2011;52:158-74.

45. Mata-Mbemba D, Iimura Y, Hazrati LN, et al. MRI, Magnetoencephalography, and surgical outcome of oligodendrocytosis versus focal cortical dysplasia type I. AJNR Am J Neuroradiol 2018;39:2371-7.

46. Ryvlin P, Cross JH, Rheims S. Epilepsy surgery in children and adults. Lancet Neurol 2014;13:1114-26.

47. Cendes F, Theodore WH, Brinkmann BH, et al. Neuroimaging of epilepsy. Handb Clin Neurol 2016;136:985-1014.

48. Shain C, Ramgopal S, Fallil Z, et al. Polymicrogyriaassociated epilepsy: a multicenter phenotypic study from the Epilepsy Phenome/Genome Project. Epilepsia 2013;54:1368-75.

49. Griffiths PD. Schizencephaly revisited. Neuroradiology 2018;60:945-60.

50. Leventer RJ, Jansen A, Pilz DT, et al. Clinical and imaging heterogeneity of polymicrogyria: a study of 328 patients.
Brain 2010;133:1415-27.

51. Pizzo F, Roehri N, Catenoix H, et al.

Epileptogenic networks in nodular heterotopia: a stereoelectroencephalography study. Epilepsia 2017;58:2112-23.

52. D'Gama AM, Geng Y, Couto JA, et al. Mammalian target of rapamycin pathway mutations cause hemimegalencephaly and focal cortical dysplasia. Ann Neurol 2015;77:720-5.

53. Kuzniecky RI. Neuroimaging of epilepsy: therapeutic implications. NeuroRx 2005;2:384-93.

54. Giulioni M, Marucci G, Martinoni M, et al. Epilepsy associated tumors: Review article. World J Clin Cases 2014;2:623-41.

55. She D, Liu J, Zeng Z, et al. Diagnostic accuracy of diffusion weighted imaging for differentiation of supratentorial pilocytic astrocytoma and pleomorphic xanthoastrocytoma. Neuroradiology 2018;60:725-33.

56. Buffa GB, Chaves H, Serra MM, et al. Multinodular and vacuolating neuronal tumor of the cerebrum (MVNT): a case series and review of the literature. J Neuroradiol 2020;47:216-20.

57. Josephson CB, Leach JP, Duncan R, et al. Seizure risk from cavernous or arteriovenous malformations: prospective population-based study. Neurology 2011;76:1548-54.

58. Morrison L, Akers A. Cerebral cavernous malformation, familial. In: Adam MP, Ardinger HH, Pagon RA, et al. editors. GeneReviews®. Seattle: University of Washington, 2003.

59. Choquet H, Pawlikowska L, Lawton MT, et al. Genetics of cerebral cavernous malformations: current status and future prospects. J Neurosurg Sci 2015;59:211-20.

60. Annegers JF, Coan SP. The risks of epilepsy after traumatic brain injury. Seizure 2000;9:453-57.

61. Temkin NR. Risk factors for posttraumatic seizures in adults. Epilepsia 2003;44:18-20.

62. Duncan JS. Selecting patients for epilepsy surgery: synthesis of data. Epilepsy Behav 2011;20:230-2.

63. Duncan JS, Winston GP, Koepp MJ, et al. Brain imaging in the assessment for epilepsy surgery. Lancet Neurol 2016;15:420-33.

64. Abbott DF, Waites AB, Lillywhite LM, et al. fMRI assessment of language lateralization: an objective approach. Neuroimage 2010;50:1446-55.

65. Rimol LM, Specht K, Hugdahl K. Controlling for individual differences in $\mathrm{fMRI}$ brain activation to tones, syllables, and words. Neuroimage 2006;30:554-62.

66. Sanjuán A, Bustamante JC, Forn C, et al. Comparison 
of two fMRI tasks for the evaluation of the expressive language function. Neuroradiology 2010;52:407-15.

67. Wray CD, Blakely TM, Poliachik SL, et al. Multimodality localization of the sensorimotor cortex in pediatric patients undergoing epilepsy surgery. J Neurosurg Pediatr 2012;10:1-6.

68. Winston GP. Epilepsy surgery, vision, and driving: what has surgery taught us and could modern imaging reduce the risk of visual deficits? Epilepsia 2013;54:1877-88.

69. Yogarajah M, Focke NK, Bonelli S, et al. Defining Meyer's loop-temporal lobe resections, visual field deficits and diffusion tensor tractography. Brain 2009;132:1656-68.

70. Piper RJ, Yoong MM, Kandasamy J, et al. Application of diffusion tensor imaging and tractography of the optic radiation in anterior temporal lobe resection for epilepsy: a systematic review. Clin Neurol Neurosurg 2014;124:59-65.

71. Jeong JW, Asano E, Juhász C, et al. Quantification of primary motor pathways using diffusion MRI tractography and its application to predict postoperative motor deficits in children with focal epilepsy. Hum Brain Mapp 2014;35:3216-26.

72. Sarikaya I. Pet studies in epilepsy. Am J Nucl Med Mol Imaging 2015;5:416-30.

73. Rathore C, Dickson JC, Teotónio R, et al. The utility of 18F-fluorodeoxyglucose PET (FDG PET) in epilepsy surgery. Epilepsy Res 2014;108:1306-14.

74. Van Paesschen W, Dupont P, Van Driel G, et al. SPECT perfusion changes during complex partial seizures in patients with hippocampal sclerosis. Brain 2003;126:1103-11.

75. Grouiller F, Thornton RC, Groening K, et al. With or without spikes: localization of focal epileptic activity by simultaneous electroencephalography and functional

Cite this article as: De Vito A, Mankad K, Pujar S, Chari A, Ippolito D, D'Arco F. Narrative review of epilepsy: getting the most out of your neuroimaging. Transl Pediatr 2021;10(4):1078-1099. doi: 10.21037/tp-20-261 magnetic resonance imaging. Brain 2011;134:2867-86.

76. van Graan LA, Lemieux L, Chaudhary UJ. Methods and utility of EEG-fMRI in epilepsy. Quant Imaging Med Surg 2015;5:300-12.

77. Thornton R, Vulliemoz S, Rodionov R, et al. Epileptic networks in focal cortical dysplasia revealed using electroencephalography-functional magnetic resonance imaging. Ann Neurol 2011;70:822-37.

78. Chaudhary UJ, Carmichael DW, Rodionov R, et al. Mapping preictal and ictal haemodynamic networks using video-electroencephalography and functional imaging. Brain 2012;135:3645-63.

79. Federico P, Abbott DF, Briellmann RS, et al. Functional MRI of the pre-ictal state. Brain 2005;128:1811-7.

80. Gotman J, Grova C, Bagshaw A, et al. Generalized epileptic discharges show thalamocortical activation and suspension of the default state of the brain. Proc Natl Acad Sci U S A 2005;102:15236-40.

81. Singh SP. Magnetoencephalography: basic principles. Ann Indian Acad Neurol 2014;17:S107-12.

82. David O, Blauwblomme T, Job AS, et al. Imaging the seizure onset zone with stereo-electroencephalography. Brain 2011;134:2898-911.

83. De Almeida AN, Olivier A, Quesney F, et al. Efficacy of and morbidity associated with stereoelectroencephalography using computerized tomography--or magnetic resonance imaging-guided electrode implantation. J Neurosurg 2006;104:483-7.

84. Garcia-Lorenzo B, Del Pino-Sedeno T, Rocamora R, et al. Stereoelectroencephalography for refractory epileptic patients considered for surgery: systematic review, meta-analysis, and economic evaluation. Neurosurgery 2019;84:326-38. 